\title{
Circle Patterns on Singular Surfaces
}

\author{
Jean-Marc Schlenker
}

Received: 6 June 2006 / Revised: 28 June 2007 /

Published online: 11 December 2007

(C) Springer Science+Business Media, LLC 2007

\begin{abstract}
We consider "hyperideal" circle patterns, i.e., patterns of disks appearing in the definition of the weighted Delaunay decomposition associated with a set of disjoint disks, possibly with cone singularities at the centers of those disks. Hyperideal circle patterns are associated with hyperideal hyperbolic polyhedra. We describe the possible intersection angles and singular curvatures of those circle patterns on Euclidean or hyperbolic surfaces with cone singularities. This is related to results on the dihedral angles of ideal or hyperideal hyperbolic polyhedra. The results presented here extend those in Schlenker (Math. Res. Lett. 12(1), 82-112, 2005), however, the proof is completely different (and more intricate) since Schlenker (Math. Res. Lett. 12(1), $82-112,2005)$ used a shortcut which is not available here.
\end{abstract}

Résumé On considère des motifs de cercles "hyperidéaux", i.e. ceux qui apparaissent dans la définition de la décomposition Delaunay pondérée associée à un ensemble de disques disjoint, éventuellement avec des singularités coniques aux centre de ces disques. Les motifs de cercles hyperidéaux sont associés aux polyèdres hyperboliques hyperidéaux. On décrit les angles d'intersection et les courbures singulières possibles de ces motifs de cercles, sur les surfaces euclidiennes ou hyperboliques à singularités coniques. C'est lié à des résultats sur les angles dièdres des polyèdres hyperboliques idéaux ou hyperidéaux. Les résultats présentés ici étendent ceux de Schlenker (Math. Res. Lett. 12(1), 82-112, 2005), mais les preuves sont complètement différentes (et plus élaborés) car Schlenker (Math. Res. Lett. 12(1), 82-112, 2005) prenait un racourçi qui n'est pas utilisable ici.

The author would like to thank the RIP program at Oberwolfach, where part of the research presented here was conducted. Partially supported by the "ACI Jeunes Chercheurs" Métriques privilégiés sur les variétés à bord, 2003-06, and the ANR program Representations of surface groups, 2007-09.

J.-M. Schlenker $(\bowtie)$

Institut de Mathématiques de Toulouse, UMR CNRS 5219, Université Paul Sabatier,

31062 Toulouse Cedex 9, France

e-mail: schlenker@math.ups-tlse.fr 
Keywords Circle patterns $\cdot$ Hyperbolic geometry $\cdot$ Hyperideal polyhedra

\section{Introduction and Results}

\subsection{Motivations}

Delaunay Decompositions Let $x_{1}, \ldots, x_{n} \in \mathbb{R}^{2}$ be points, and suppose that the convex hull $C$ of those points has a nonempty interior. There is then a unique Delaunay decomposition of $C$ : a decomposition of $C$ as the union of convex polygons with vertices at the $x_{i}$ such that, for each face $f$, there is a circle $C_{f}$ which contains exactly the $x_{i}$ which are vertices of $f$ but whose interior contains none of the $x_{i}$.

The same construction can be made in the sphere (with at least three points) or in a hyperbolic surface. The circles appearing in this definition, with their peculiar types of intersections, are forming what is defined in the following as an ideal circle pattern. Ideal circle patterns are related in a natural way to ideal hyperbolic polyhedra.

The possible intersection angles of ideal circle patterns can be described in terms of some linear equalities and inequalities. This was done for the sphere (in the context of ideal hyperbolic polyhedra) by Andreev [2] and Rivin [31]. Related results were obtained on the sphere by Luo [25], and on higher genus surfaces by different authors [8, 17, 23, 30, 32]; in particular, Bobenko and Springborn [4] recently gave a statement, for hyperbolic surfaces, which is closely related to Theorem 1.6 below. A similar result is also stated in [38].

Delaunay Decompositions with Singular Points Suppose now that instead of being simply points on an Euclidean (or hyperbolic) surface $S, x_{1}, \ldots, x_{n}$ are conical singularities. It remains true that there exists a unique Delaunay decomposition of $S$ with vertices at the $x_{i}$. The circles that appear in the definition of this Delaunay decomposition still constitute an "ideal circle pattern", but now there are conical singularities at the intersection points of the circles. Again it is of interest to understand the possible intersection angles of the circles, and results that address this question can be found in $[4,8,17,23]$.

Weighted Delaunay Decomposition Associated to Disks Another generalization of the notion of Delaunay decomposition can be obtained by replacing the points $x_{1}, \ldots, x_{n}$ by disjoint disks $D_{1}, \ldots, D_{n}$. To such a finite set of disks, say in a Euclidean surface $S$, one can still associate in a unique way an embedded graph $\Gamma$, with vertices $v_{1}, \ldots, v_{n}$ corresponding to the $D_{i}$, such that to each face $f$ of $\Gamma$ is associated a circle $C_{f}$ which is orthogonal to the $D_{i}$ corresponding to the vertices of $f$, but either does not intersect, or intersects with an angle less than $\pi / 2$, all the other disks $D_{i}$. It is customary to describe this notion as a "weighted Delaunay decomposition"; see e.g. [15].

The circles $C_{f}$ involved in this definition constitute what is defined in the following as a "hyperideal circle pattern", see Fig. 1. Hyperideal circle patterns are naturally associated with hyperideal hyperbolic polyhedra. The $D_{i}$ are actually allowed in the definition to be points, so that ideal circle patterns are special cases of hyperideal circle patterns. 
As for ideal circle patterns, it is interesting to describe the possible intersection angles between the circles in a hyperideal circle pattern. For the sphere, a complete description is hidden behind a theorem of Bao and Bonahon [3] on hyperideal polyhedra, while statements concerning surfaces of genus at least 2 are similarly consequences of results [34] on equivariant hyperideal polyhedra. Further results can be found in $[39,40]$.

It is also possible to consider the Delaunay decomposition associated to a set of disks containing conical singularities at their centers. The main goal of this paper is to give simple statements describing the possible intersection angles of the circles in this context, when the underlying metric is either Euclidean (this is Theorem 1.4) or hyperbolic (Theorem 1.6). Those statements are new even for ideal circle patterns with conical singularities.

Circle Patterns as Descriptions of Singular Surfaces Consider a Euclidean (or possibly hyperbolic) surface with conical singularities, for instance a polyhedral surface in Euclidean space with its induced metric. Such surfaces occur in many practical applications and it is interesting to find practical ways to describe them. The most natural way is, of course, to choose a triangulation, with the conical points as vertices, and to describe the surface by the combinatorics of the triangulation and the lengths of the edges.

There is another possible way, however, based on the Delaunay decomposition associated with the conical singularities. It provides an ideal circle pattern which is uniquely determined by the singular curvature at the cone points of the metric, as well as some intersection angles between the circles. It follows from Theorem 1.4 that the singular metric can be uniquely reconstructed from this data. This idea is used in a practical manner in [20]. This description of singular metrics has some interesting characteristics:

- It gives a direct access to the curvature, since the total curvature of a domain is simply the sum of the curvatures of the singular points it contains, which is part of the description.

- It is sensitive to the conformal structure, in particular it leads directly to a "discrete conformal map" to a domain in the plane, obtained by constructing the circle patterns with the same intersection angles but with no singular curvature.

- The complete description of the possible data (for a given combinatorics) is "simple", since it is given by a set of linear inequalities between the possible intersection angles and singular curvatures.

- The computations that are needed involve the maximization of a functional under linear constraints, so they should be algorithmically simple.

It is also possible to apply the same idea, taking the Delaunay decomposition associated with disjoint disks centered at the singular points. Such a set of disks uniquely determines a hyperideal circle pattern and, again by Theorem 1.4, the surface and the radii of the disks can be recovered from the combinatorics, the singular curvatures and the intersection angles of the circles. Compared to a description by an ideal circle pattern, this description has the advantage of being more flexible, since the possible intersection angles between the circles are required to satisfy affine inequalities rather than equalities. 
Relations with Geometric Topology Circle packings, and "ideal" circle patterns, are related to three-dimensional hyperbolic manifolds, orbifolds and cone-manifolds. Given an ideal circle pattern, one can associate to it a noncomplete three-dimensional hyperbolic manifold with polyhedral boundary. Gluing two copies of these manifolds along the boundary yields a three-dimensional hyperbolic cone-manifold of finite volume, which is an orbifold when the intersection angles of the original circle pattern are of the form $\pi / k, k \geq 2$. In the same manner, "hyperideal" circle patterns naturally yield, by a similar construction, compact three-dimensional cone-manifolds (resp., orbifolds). This point will not be developed much here, but it appears in Sect. 7 as a key tool in the proof of the main results.

A Short Comment on the Proof The proof is based on the deformation method: for a given combinatorics, one considers the natural map sending a "hyperideal" circle pattern, with the right incidence graph, to the set of its intersection angles and singular curvatures. One has to prove that this map is a homeomorphism. The key point in the proof is the fact that the map has injective differential at each point, in other terms that hyperideal circle patterns are "rigid".

There are several different methods to prove this kind of rigidity. In [3], it was done using an argument going back to Legendre [22] and Cauchy [10] (see Sabitov's illuminating paper on the subject [35]). In some situations involving equivariant polyhedra (see e.g. [34]), the infinitesimal rigidity can be obtained, through a transformation due to Pogorelov [28], as consequences of similar statements for equivariant polyhedra in the Minkowski space; see [42] (similar ideas were used for smooth surfaces in [21]). The results in [40] are based on results (from [7, 27]) on the geometry of the convex core of hyperbolic three-manifolds, which in turn rely on a local rigidity result of Hogdson and Kerckhoff [19].

The proof of the infinitesimal rigidity used here, by contrast, is based on a volume argument going back to $[9,30]$ for ideal hyperbolic polyhedra. The key point, already used in $[39,41]$, is that the argument also work for hyperideal polyhedra, because their volume is, as for ideal polyhedra, a strictly concave function of their dihedral angles.

\subsection{Definitions}

Ideal Circle Packings We now move to a more precise description of the results. We define ideal circle patterns in $S^{2}$, although the definition could be given in the Euclidean or the hyperbolic plane. Ideal circle patterns are naturally associated to ideal hyperbolic polyhedra, a point which should become clear later.

Definition 1.1 A circle pattern on $S^{2}$ is a finite family of oriented circles $C_{1}, \ldots, C_{N}$. Given a circle pattern, an interstice is a connected component of the complement of the union of the open disks bounded by the circles. If $C_{1}, \ldots, C_{N}$ is a circle pattern, it is ideal if:

- each interstice is a point,

- each circle contains at least three interstices, and

Springer 
- if $D$ is an open disk in $S^{2}$, containing no interstice, but such that its closure contains at least three of the interstices, then $D$ is the open disk bounded by one of the $C_{i}, 1 \leq i \leq N$.

Given an ideal circle pattern, its incidence graph is the graph, embedded in the sphere which has:

- one vertex for each circle, and

- one edge between two vertices, when the corresponding circles intersect at two interstices.

Clearly, each ideal circle pattern is the pattern of circles appearing in the definition of the Delaunay decomposition of a set of points, namely its interstices. Notice that, with the definition given here, two circles can intersect while the corresponding vertices of the incidence graph are not adjacent.

Again, the definition can clearly be extended from the sphere to any hyperbolic or Euclidean surface. Results concerning the possible intersection angles of ideal circle patterns can be found in $[4,23,32,38]$.

Hyperideal Circle Patterns There is a related notion of "hyperideal" circle patterns, which are related to hyperideal hyperbolic polyhedra as ideal circle patterns are related to ideal hyperbolic polyhedra, see Fig. 1. Given a circle pattern, the intersection angle between two intersecting circles is $\pi$ minus the angle measured in the intersection of the disks bounded by the two circles. In other terms, this angle is measured in the complement of one of the disks in the other. This angle is defined to be equal to $\pi$ when the circles are tangent.

Definition 1.2 Let $C_{1}, \ldots, C_{N}$ be a circle pattern in $S^{2}$, with interstices $I_{1}, \ldots, I_{M}$. It is hyperideal if:

- Each interstice either is a point, or is topologically a disk.

- Each circle intersects at least three interstices.

- For each $j \in\{1, \ldots, M\}$, corresponding to an interstice which is not a point, there is an oriented circle $C_{j}^{\prime}$, containing $I_{j}$, which is orthogonal to all the circles $C_{i}$ adjacent to $I_{j}$. If $I_{j}$ is a point we set $C_{j}^{\prime}:=I_{j}$, for all $i \in\{1, \ldots, N\}$ and all $j \in$ $\{1, \ldots, M\}$, if $C_{i}$ is not adjacent to $I_{j}$, then either the interior of $C_{i}$ is disjoint from $C_{j}^{\prime}$, or $C_{i}$ intersects $C_{j}^{\prime}$ (which is not a point) and their intersection angle is strictly larger than $\pi / 2$.

- If $D$ is an open disk in $S^{2}$ such that:

1. for each $j \in\{1, \ldots, M\}$, either $D$ is disjoint from $C_{j}^{\prime}$, or $\partial D$ has an intersection angle at least $\pi / 2$ with $C_{j}^{\prime}$, and

2. there are at least three $C_{j}^{\prime}$ which are either points contained in $\partial D$ or circles orthogonal to $\partial D$,

then $\partial D$ is one of the $C_{i}$.

Such a pattern is strictly hyperideal if no interstice is reduced to a point. The circles $C_{i}$ are called principal circles, while the circles (or points) $C_{j}^{\prime}$ are the dual circles. 
Fig. 1 A hyperideal circle pattern and its incidence graph (the dual circles are dashed)

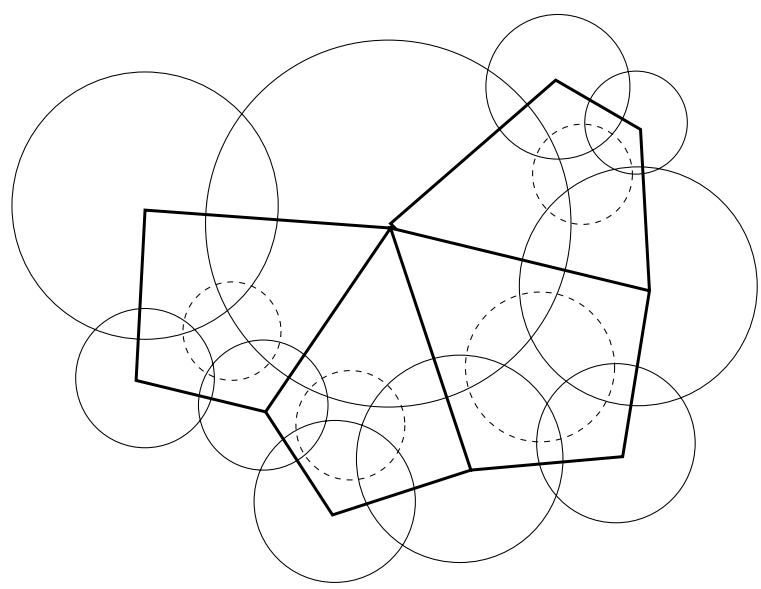

The incidence graph of a hyperideal circle pattern is defined as for ideal circle patterns in Definition 1.1. Hyperideal circle patterns have a simple relation to circle packings: those correspond simply to the limit when all intersection angles go to $\pi$.

Clearly the notion of circle pattern-and the notion of angles between the circles-is not limited to spherical metrics; one could also consider Euclidean or hyperbolic metrics. Actually one could simply consider a complex projective structure, also called a $\mathbb{C} P^{1}$-structure. This viewpoint is often interesting when thinking about circle patterns; however, it will not appear much here.

Some results concerning the possible intersection angles of hyperideal circle patterns can be found in [39, 40] and (in the related setting of hyperideal hyperbolic polyhedra) in $[3,34]$. Our main goal here is to extend them to singular surfaces.

Given a hyperideal circle pattern, there are actually two families of circles that one can consider. The first is made of the principal circles, appearing directly in the circle pattern; the other contains the dual circles, associated to the faces of the incidence graph, which are orthogonal to the circles of the first family. Those dual circles are disjoint, but they also provide a circle pattern of another kind, which is also of some interest, although this point will not be pursued here.

Circle Patterns on Singular Surfaces We have already mentioned that the object of this paper is to extend results on hyperideal circle patterns to Euclidean or hyperbolic surfaces with conical singularities. But let $\Sigma_{0}$ be a complete Euclidean or hyperbolic surface with one conical singularity. It is then not difficult to check that the interior of a circle on $\Sigma_{0}$ cannot contain the singular point, except at its center-otherwise the circle would not "close up".

It follows that hyperideal circle patterns can be considered only on surfaces with conical singularities if the singularities are at the centers of the principal or the dual circles. We consider here only the second possibilities, i.e. singularities at the centers of the dual circles. More general results could perhaps be obtained by also considering singularities at the centers of the principal circle, however, it would be at the cost of more complicated notations and of some added technical difficulties. It is interesting to note, however, that some of the technical points discussed in the proofs of the main results extend to this more general situation. 
Fig. 2 An admissible domain and its boundary

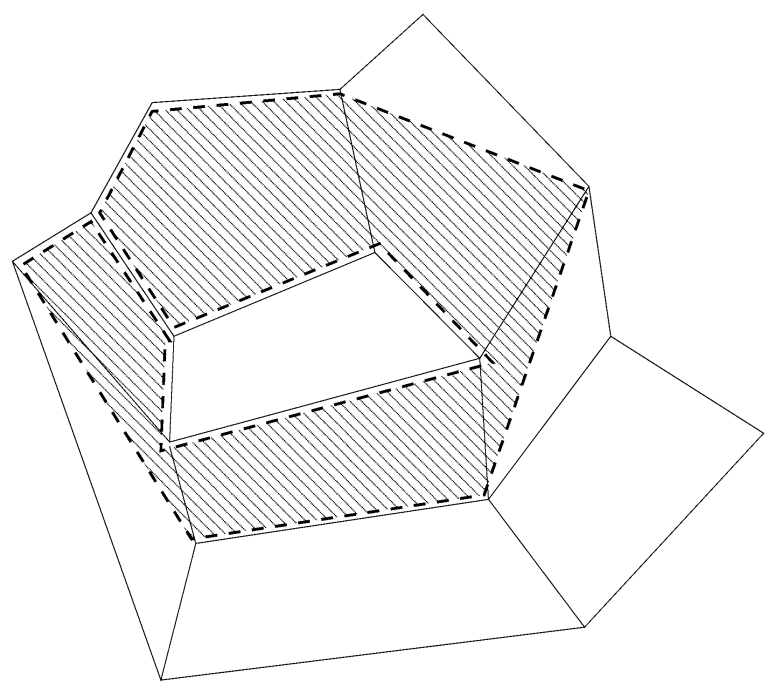

\subsection{Main Results}

Admissible Domains We need one more definition, depicted in Fig. 2, before giving the first result of this text. Let $\Gamma$ be a graph embedded in a closed surface $\Sigma$.

Definition 1.3 An admissible domain in $(\Sigma, \Gamma)$ is a connected open domain $\Omega$, which contains an open face of $\Gamma$, such that $\partial \Omega$ is a finite union of segments which:

- have as endpoints vertices of $\Gamma$, and

- either are edges of $\Gamma$ or are contained (except for their endpoints) in an open face of $\Gamma$.

To each such admissible domain, we can associate two numbers: its Euler characteristic, $\chi(\Omega)$, and the number of boundary segments contained in open faces of $\Gamma$, $m(\Omega)$. The boundary of $\Omega$ will not be understood in the usual way, but rather as the parameterized curve immersed in $\Sigma$ as the boundary of $\Omega$, considered as the image of an embedding of a surface with boundary. For instance, edges of $\Gamma$ which are not contained in $\Omega$ but are bounding two faces contained in $\Omega$ are considered as contained twice in $\partial \Omega$.

In other terms, the boundary of $\Omega$ is contained in $\Gamma$, except for some segments of $\partial \Omega$ which are contained in faces of $\Gamma$. The definition of the boundary of $\Omega$ should be compared with similar considerations in [4].

Main Results on Closed Surfaces The first result describes hyperideal circle patterns on a closed surface with a singular Euclidean metric. Given a graph $\Gamma$, we denote by $\Gamma_{1}$ the set of its edges, and by $\Gamma_{2}$ the set of its faces.

Theorem 1.4 Let $\Sigma$ be a closed orientable surface, and let $\Gamma$ be the one-skeleton of a cellular decomposition of $\Sigma$. Let $\kappa: \Gamma_{2} \rightarrow(-\infty, 2 \pi)$ and $\theta: \Gamma_{1} \rightarrow(0, \pi)$ be 
two functions. There exists a flat metric $h$ with conical singularities on $\Sigma$, with a hyperideal circle pattern $\sigma$ with incidence graph $\Gamma$, intersection angles given by $\theta$, and singular points of curvatures given by $\kappa$ at the centers of the dual circles, if and only if:

1. $\sum_{f \in \Gamma_{2}} \kappa(f)=2 \pi \chi(\Sigma)$, and

2. for any admissible domain $\Omega \subset \Sigma$

$$
\sum_{e \in \Gamma_{1}, e \subset \partial \Omega} \theta(e) \geq(2 \chi(\Omega)-m(\Omega)) \pi-\sum_{f \in \Gamma_{2}, f \subset \Omega} \kappa(f),
$$

and the inequality is strict unless $\Omega$ is a face of $\Gamma$.

The metric $h$ is then unique up to homotheties, and $\sigma$ is unique given $h$.

The sum over the edges of $\Gamma$ which are in $\partial \Omega$ should be understood as mentioned earlier, $\partial \Omega$ is considered as a parameterized immersed polygonal curve, and the edges of $\Gamma$ in $\partial \Omega$ which bound two faces in $\Omega$ are thus counted twice.

Remark 1.5 Given $(\Sigma, \Gamma)$, there is a finite number of possible admissible domains. There is therefore a finite number of inequalities of type (2) to check when applying Theorem 1.4.

Note that, in Theorem 1.4, the flat metric on $\Sigma$ is not fixed: it is "chosen" by the combinatorics of $\Gamma$ and by the functions $\theta$ and $\kappa$. The cone singularities mentioned here are at the centers of the dual circles, with a cone singularity of singular curvature $\kappa(f)$ at the center of the dual circle corresponding to the face $f$ of $\Gamma$. It is interesting to note that this result can be formulated in a simpler manner under some more restricted conditions on the conical singularities, in particular when there is no singularity at all, or when $\kappa(f) \geq 0$ for all faces $f$ of $\Gamma$; then it is sufficient to consider admissible domains $\Omega$ such that $\chi(\Omega) \geq 1$, i.e. disks, and that $m(\Omega) \leq 1$. The case with no singular points is treated in [40], while [4] considers singular surfaces, but in the restricted context of "ideal" circle patterns, and contains a slightly different statement.

Even under the general conditions for which they are stated, the hypotheses are not as complicated as they first appear. It is not too difficult, computationally speaking, to enumerate the possible domains on which condition (2) has to be checked; for most of those domains, the right-hand side of the equation in condition (2) is likely to be negative, so that the condition is trivially satisfied.

A similar result holds for higher genus orientable closed surfaces, with singular hyperbolic metrics.

Theorem 1.6 Let $\Sigma$ be a closed orientable surface, and let $\Gamma$ be the one-skeleton of a cellular decomposition of $\Sigma$. Let $\kappa: \Gamma_{2} \rightarrow(-\infty, 2 \pi)$ and $\theta: \Gamma_{1} \rightarrow(0, \pi)$ be two functions. There exists a hyperbolic metric $h$ with conical singularities on $\Sigma$, with a hyperideal circle pattern $\sigma$ with incidence graph $\Gamma$, intersection angles given by $\theta$, and singular curvatures given by $\kappa$, if and only if:

1. $\sum_{f \in \Gamma_{2}} \kappa(f)>2 \pi \chi(\Sigma)$, and 
2. for any admissible domain $\Omega \subset \Sigma$

$$
\sum_{e \in \Gamma_{1}, e \subset \partial \Omega} \theta(e) \geq(2 \chi(\Omega)-m(\Omega)) \pi-\sum_{f \in \Gamma_{2}, f \subset \Omega} \kappa(f),
$$

and the inequality is strict unless $\Omega$ is a face of $\Gamma$.

$h$ and $\sigma$ are then unique.

Simpler Examples As in the case of flat metrics, the statements become much simpler when one considers nonsingular metrics, or more generally only conical points with positive singular curvature. Indeed under this hypothesis the right-hand side in the second condition is positive only for disks with at most one boundary component in a face of $\Gamma$. This explains the simpler form of the statements obtained e.g. in [3] or in [4].

Surfaces with Geodesic Boundary There is natural extension of Theorem 1.4 and Theorem 1.6 to flat or hyperbolic surfaces with geodesic boundary. To do so, it is necessary to extend slightly the definitions given earlier. We consider a compact surface with boundary, $\Sigma$, along with a graph $\Gamma$ embedded into $\Sigma$ so that the union of the closures of the faces of $\Gamma$ covers $\Sigma$. In other terms, the boundary of $\Sigma$ is covered by edges and vertices of $\Sigma$. We also extend the definition of an admissible domain, as in Definition 1.3.

Definition 1.7 Let $\Gamma$ be a graph embedded in a compact surface with boundary $\Sigma$, such that $\partial \Sigma \subset \Gamma$. An admissible domain in $(\Sigma, \Gamma)$ is a domain $\Omega \subset \Sigma$, containing a face of $\Gamma$, such that $\partial \Omega$ is a finite union of segments which:

- have as endpoints vertices of $\Gamma$, and

- either are edges of $\Gamma$, or have interior contained in a face of $\Gamma$.

We define $\chi(\Omega)$ as the Euler characteristic of $\Omega$, and $m(\Omega)$ as the number of maximal segments in $\partial \Omega$ which are contained either in a face of $\Gamma$ or in $\partial \Sigma$ (i.e. this excludes connected components of $\partial \Sigma$ which are boundary components of $\partial \Omega$, because they are closed curves rather than segments).

There is a natural notion of circle pattern with incidence graph $\Gamma$, on $\Sigma$ endowed with a hyperbolic (resp. Euclidean) metric with geodesic boundary. Such a circle pattern has one circle for each interior vertex of $\Gamma$, and, for each boundary vertex of $\Gamma$, a half-circle, which intersects orthogonally the boundary of $\Sigma$.

Theorem 1.8 Let $\Sigma$ be a compact orientable surface with boundary. Let $\Gamma$ be the one-skeleton of a cellular decomposition of $\Sigma$, let $\kappa: \Gamma_{2} \rightarrow(-\infty, 2 \pi)$ and let $\theta: \Gamma_{1} \rightarrow(0, \pi)$ be two functions. There exists a hyperbolic metric $h$ with conical singularities on $\Sigma$, with a hyperideal circle pattern $\sigma$ with incidence graph $\Gamma$, intersection angles given by $\theta$, and singular curvatures given by $\kappa$, if and only if:

1. $\sum_{f \in \Gamma_{2}} \kappa(f)>2 \pi \chi(\Sigma)$, and 
2. for any admissible domain $\Omega \subset \Sigma$

$$
\sum_{e \subset \partial \Omega \backslash \partial \Sigma} \theta(e) \geq \pi(2 \chi(\Omega)-m(\Omega))-\sum_{f \subset \Omega} \kappa(f),
$$

with strict inequality except perhaps when $\Omega$ is a face of $\Gamma$.

$h$ and $\sigma$ are then unique.

This theorem is a special case — which is more readily understandable - of Theorem 1.11. A similar statement can be given for Euclidean metrics with geodesic boundary, based on Theorem 1.10, we leave this point to the interested reader.

Framed Circle Patterns Theorem 1.8 is not completely satisfactory, in particular because it does not apply to circle patterns in the plane (even without singularities). This paragraph contains more flexible statements, which remain quite simple but for which some additional definitions are necessary.

We first give the definition for a disk, so as to simplify the notation a little (see Fig. 3). A more general definition is used in the following, but the only difference is in the number of boundary components.

Definition 1.9 Let $D$ be a closed disk with a Euclidean metric with cone singularities, and with piecewise geodesic boundary. Let $g_{1}, g_{2}, \ldots, g_{n}$ be the geodesic segments in $\partial D$, in the cyclic order in which they appear. A framed circle pattern on $D$ is a hyperideal circle pattern, with singularities at the center of the dual circles, with incidence graph $\Gamma$ (considered as a graph embedded in $D$ ) so that:

- $\Gamma$ has $n$ boundary vertices, corresponding to circles $C_{1}, \ldots, C_{n}$ (in the cyclic order of the boundary vertices of $\Gamma$ );

- for each $i=1, \ldots, n$, the circle $C_{i}$ intersects the interior of the segment $g_{i}$ in two points; and

- for each $i \in \mathbb{Z} / n \mathbb{Z}$, there is a circle centered at the intersection point of $g_{i}$ and $g_{i+1}$ which is orthogonal to both $C_{i}$ and $C_{i+1}$.

Note that in the second condition the intersection of the boundary segments with the corresponding circles are not at the endpoints of the segments, in other terms the "dual" circles centered at the vertices of the boundary polygon are not reduced to points.

Given a framed circle pattern, the intersection angles between the oriented geodesic segments $g_{i}$ and $g_{i+1}$, for $i=1, \ldots, n$, are called its polygonal angles, they are equal to $\pi$ minus the angle at the intersection point of the interior of the polygon with edges the $g_{i}$. The intersection angles between $g_{i}$ and $C_{i}$, for $i=1, \ldots, n$, are called the boundary angles, they are defined as the angle at the intersection point of the domain of the interior of $C_{i}$ which is outside the polygon with edges the $g_{i}, i=1, \ldots, n$.

The Extended Graph of a Framed Circle Pattern It is also convenient to consider combinatorial data that are slightly more elaborate than the incidence graph $\Gamma$ of the circle pattern. The extended incidence graph $\Gamma^{\prime}$ of a framed circle pattern has, in addition to the incidence graph $\Gamma$ : 
Fig. 3 A framed hyperideal circle pattern

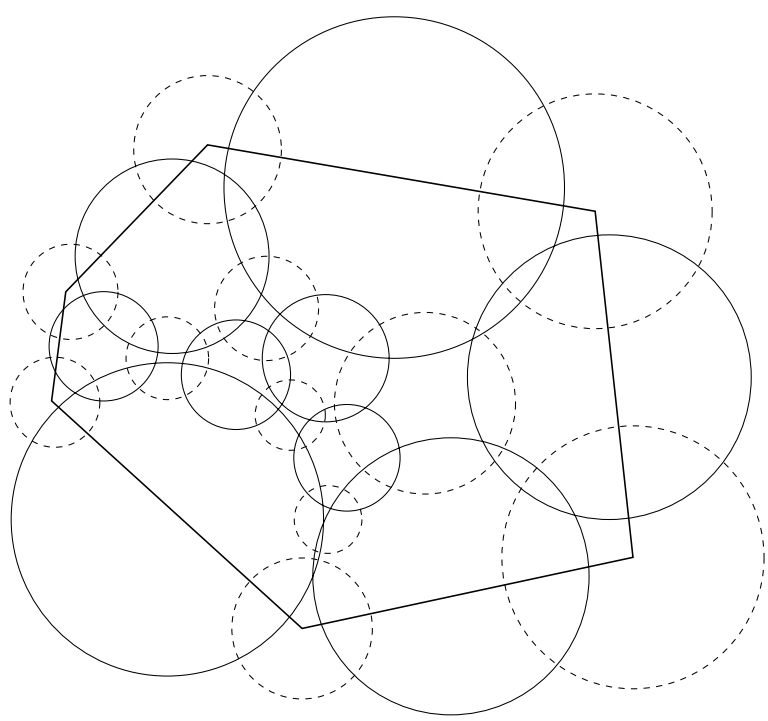

- one vertex for each of the $g_{i}, i=1, \ldots, n$; those are called the boundary vertices of $\Gamma^{\prime}$, and they form a set denoted by $\Gamma_{0,2}^{\prime}$;

- one edge going from the vertex corresponding to $g_{i}$ to the vertex corresponding to $g_{i+1}$, for each $i=1, \ldots, n$, those edges also form a set denoted by $\Gamma_{1, \partial}^{\prime}$; and

- one edge going from the vertex corresponding to $C_{i}$ to the vertex corresponding to $g_{i}$, for each $i=1, \ldots, n$.

Clearly $\Gamma^{\prime}$ depends only on $\Gamma$ (and not on other data from the framed circle pattern) so we will call $\Gamma^{\prime}$ the extended graph of $\Gamma$.

Since $\Gamma^{\prime}$ is not embedded in a closed surface but in a "surface with boundary", it is relevant to define what is meant by the dual graph $\Gamma^{* *}$ : it is the graph which has one vertex for each face of $\Gamma^{\prime}$, one edge between two vertices of $\Gamma^{\prime *}$ when the corresponding faces of $\Gamma^{\prime}$ are adjacent (in particular there is no edge corresponding to the boundary edges of $\Gamma^{\prime}$ ), and one face for each vertex of $\Gamma$ (i.e. not for the boundary vertices of $\Gamma^{\prime}$ ).

Just as the edges of $\Gamma$ carry naturally a "weight", which is the intersection angle between the corresponding circles of the pattern, the additional edges of $\Gamma^{\prime}$ carry natural weights:

- For the edge going from the vertex corresponding to $g_{i}$ to the vertex corresponding to $g_{i+1}$, it is the "polygonal" angle between $g_{i}$ and $g_{i+1}$, as defined earlier.

- For the edge going from the vertex corresponding to $C_{i}$ to the vertex corresponding to $g_{i}$, it is the "boundary" angle between $C_{i}$ and $g_{i}$.

We call these data the "extended intersection angles" of the circle pattern. The considerations made here are of course not limited to topological disks, one can also define in the same way framed circle patterns on any compact surface with boundary.

One additional notation is necessary: given a compact surface with boundary $\Sigma$ and a graph $\Gamma$ embedded in $\Sigma$, and given an admissible domain $\Omega$ in $\left(\Sigma, \Gamma^{\prime}\right)$, we 
denote by $n(\Omega)$ the number of segments in $\partial \Omega \cap \partial \Sigma$. Note that the term "segment" excludes boundary components which are topologically circles.

We can now state an analog of Theorem 1.4 for framed circle patterns on singular Euclidean surfaces with boundary.

Theorem 1.10 Let $\Sigma$ be compact orientable surface with boundary. Let $\Gamma$ be the one-skeleton of a cellular decomposition of $\Sigma$, and let $\Gamma^{\prime}$ be the extended graph of $\Gamma$. Let $\kappa: \Gamma_{2} \rightarrow(-\infty, 2 \pi)$, let $\theta: \Gamma_{1}^{\prime} \rightarrow(0, \pi)$ be functions. There exists an Euclidean metric $h$ with conical singularities on $\Sigma$, with a hyperideal circle pattern $\sigma$ with incidence graph $\Gamma$, extended intersection angles given by $\theta$, and singular curvatures given by $\kappa$, if and only if:

1. $\sum_{f \in \Gamma_{2}} \kappa(f)=2 \pi \chi(\Sigma)-\sum_{v \in \Gamma_{1, \partial}^{\prime}} \theta(v)$, and

2. for any admissible domain $\Omega$ in $\left(\Sigma, \Gamma^{\prime}\right)$

$$
\sum_{e \in \Gamma_{1}^{\prime}, e \in \partial \Omega} \theta(e) \geq(2 \chi(\Omega)-m(\Omega)-n(\Omega)) \pi-\sum_{f \in \Gamma_{2}, f \subset \Omega} \kappa(f),
$$

with strict inequality except that:

- equality is possible when $\Omega$ is a face of $\Gamma$,

- equality is required when $\Omega$ is the union of all faces of $\Gamma$ (except the exterior face).

$h$ is then unique up to homotheties, and $\sigma$ is unique.

Note that the second exception in condition (2) is a reformulation of condition (1), we keep the two conditions for the sake of unity with the other similar statements. There is a similar result for framed hyperbolic circle patterns.

Theorem 1.11 Let $\Sigma$ be a compact orientable surface with nonempty boundary. Let $\Gamma$ be the one-skeleton of a cellular decomposition of $\Sigma$, and let $\Gamma^{\prime}$ be the extended graph of $\Gamma$. Let $\kappa: \Gamma_{2} \rightarrow(-\infty, 2 \pi)$, let $\theta: \Gamma_{1}^{\prime} \rightarrow(0, \pi)$ be functions. There exists a hyperbolic metric $h$ with conical singularities on $\Sigma$, with a hyperideal circle pattern $\sigma$ with incidence graph $\Gamma$, extended intersection angles given by $\theta$, and singular curvatures given by $\kappa$, if and only if:

1. $\sum_{f \in \Gamma_{2}} \kappa(f)>2 \pi-\sum_{v \in \Gamma_{1, \partial}} \theta(v)$, and

2. for any admissible domain $\Omega$ in $\left(\Sigma, \Gamma^{\prime}\right)$

$$
\sum_{e \in \partial \Omega} \theta(e) \geq(2 \chi(\Omega)-m(\Omega)-n(\Omega)) \pi-\sum_{f \subset \Omega} \kappa(f),
$$

with strict inequality except perhaps when $\Omega$ is a face of $\Gamma$.

$h$ and $\sigma$ are then unique.

When $\kappa \equiv 0$ and $\Sigma$ is a disk, both Theorem 1.10 and Theorem 1.11 are direct consequences of a theorem of Bao and Bonahon [3] on the dihedral angles of hyperideal polyhedra. 


\subsection{Another Possible Approach}

Some of the results presented here, in particular those concerning hyperideal circle patterns on Euclidean surfaces, can also be obtained in a different way, replacing the deformation approach favored here by a direct study of the critical points of some volume-based functionals. This is much more explicit, or even constructive, than the approach followed here, and it is better suited to a computer implementation. On the other hand, the proof given here is perhaps more flexible.

The second method of proof, based more directly on singular points of volumebased functionals, was followed recently by Boris Springborn [43], leading to different but strongly related results on Euclidean circle patterns. Springborn considered a triangulation of a surface (possibly with boundary), which corresponds to the dual of the incidence graph of the circle pattern which we consider here, along with a number $\theta(e) \in(0, \pi)$ for each edge $e$ and another number $\kappa(v) \in(-\infty, 2 \pi)$ for each vertex $v$. He then defined a coherent angle system as the attribution, to each triangle, of six numbers (one for each edge and one for each vertex) which satisfy some linear equalities and inequalities, in particular the sum of the two numbers attributed to each edge $e$ (one for each triangle containing it) is equal to $\theta(e)$, while the sum of the numbers attached to vertex $v$ is equal to $\kappa(v)$. He then proved that the numbers $\theta$ and $\kappa$ can be realized as the intersection angles and singular curvatures of a hyperideal circle pattern on a Euclidean surface with cone singularities if and only if a coherent angle system exists.

It follows from both Springborn's results and Theorem 1.4 here that a coherent angle system exists if and only if the conditions of Theorem 1.4 are satisfied. Once this is established for given data (incidence graph, intersection angles and singular curvatures) the ideas developed by Springborn [43] might be used to actually construct the hyperideal circle pattern using an algorithm based on the maximization of a functional under linear constraints.

The results presented here are actually to a large extent the result of a collaboration with Boris Springborn, whose contribution is important. We decided, however, to write two papers, each taking an approach toward which he was inclined.

\section{Outline of the Proof}

Since the proofs of the main results are somewhat intricate and have some technical parts, it appears helpful to give first a brief outline of the way they proceed.

Theorem 1.10 follows from Theorem 1.4, and Theorem 1.11 from Theorem 1.6, so we concentrate on those statements here.

\subsection{A Deformation Method}

The proof of the two main results follow a so-called deformation method, which has been classical at least since the work of Aleksandrov [1] on convex polyhedra. First we choose a graph $\Gamma$ embedded in a closed surface. Then we consider a space $\mathcal{C}(\Gamma)$ of circle patterns with combinatorics given by $\Gamma$, and a space $\mathcal{D}(\Gamma)$ of intersection data and singular curvatures at the singular points satisfying the hypothesis of the 
theorem. There is also a natural map $\Phi_{\Gamma}$ defined on $\mathcal{C}(\Gamma)$ sending a circle pattern to its intersection angles and singular curvatures. It is then necessary to check the following points:

1. The image of $\Phi_{\Gamma}$ is contained in $\mathcal{D}(\Gamma)$, i.e. the conditions in the theorem are necessary.

2. The spaces $\mathcal{C}(\Gamma)$ and $\mathcal{D}(\Gamma)$ are differentiable manifolds of the same dimension.

3. The map $\Phi_{\Gamma}$ has injective differential at each point of $\mathcal{C}(\Gamma)$. This can be formulated as an infinitesimal rigidity statement: it is not possible to deform infinitesimally a circle pattern without changing either the intersection angles or the singular curvatures.

4. $\Phi_{\Gamma}$ is proper. This translates as a compactness property: if a sequence of circle patterns is such that the intersection angles and the singular curvatures converge to a "good" limit, than it has a converging subsequence.

5. $\mathcal{C}(\Gamma)$ is nonempty, $\mathcal{D}(\Gamma)$ is connected, and each element of $\mathcal{D}(\Gamma)$ has at most one inverse image by $\Phi_{\Gamma}$.

Point (1) is mostly elementary, and is proved in Sect. 3. Point (3) is perhaps the key part of the proof, and is done-for each of the situations considered-in Sect. 5. Points (2) and (5) are proved as consequences of the methods developed there, with point (5) proved in Sect. 7. Point (4) also demands some efforts, it is treated in Sect. 6.

It is well known, and the consequence of simple topological arguments, that points (1) to (4) imply that $\Phi_{\Gamma}$ is a covering of $\mathcal{D}(\Gamma)$ by $\mathcal{C}(\Gamma)$. In other terms, for each choice of $\Gamma$, there is an integer $N_{\Gamma} \in \mathbb{N}$ such that each point of $\mathcal{D}(\Gamma)$ has exactly $N_{\Gamma}$ inverse images by $\Phi_{\Gamma}$. It follows from point (5) that $N_{\Gamma}$ is at most 1 . It remains to prove that $N_{\Gamma}=1$ for any choice of $\Gamma$.

We show in Sect. 7 that, for Theorem 1.6, this follows from important results in geometric topology, in particular the Orbifold Hyperbolization Theorem [6]. To show the same result for Euclidean surfaces, we use in Sect. 7 an approximation argument, based on another use of our compactness statement, Lemma 6.1, which permits us to obtain a circle pattern on a Euclidean surface with conical singularities as a limit of circle patterns on hyperbolic surfaces (after multiplication of the hyperbolic metrics by some coefficients).

We can now consider with a little more detail the main points of the proof, in particular points (3) and (4) of the outline above.

\subsection{Compactness}

This part can be found in Sect. 6. There are different ways to prove the kind of compactness property needed here, in particular in the context of hyperbolic polyhedra (see e.g. [11, 33, 36, 37]) or ideal circle patterns on singular surfaces (see e.g. [4, 30]). However, the condition appearing in the main statements here, concerning linear inequalities for each admissible domain, is more elaborate than the condition in earlier references, which concerned only topological disks, and this is partly reflected in the fact that the compactness argument is also a little more elaborate. The approach followed here, however, is elementary, without reference to the underlying three-dimensional hyperbolic geometry, and only in terms of circle patterns. 


\subsection{Hyperbolic Polyhedra and Circle Patterns}

As the reader might have already well understood, the results on circle patterns presented here rely heavily on tools from three-dimensional hyperbolic geometry, and in particular on some properties of the volume of hyperideal hyperbolic polyhedra. This is developed in Sect. 4, and we give a short outline here. The ideas go back to hyperbolic geometry construction for circle packings (in particular [9, 12, 44]) and for "ideal" circle patterns (see in particular [4, 23, 30]).

Let's first describe the topological aspects of the construction. Given an embedded graph on a surface $\Sigma$ we define a three-dimensional cell complex $\mathcal{S}$, which is a cone over the graph $\Gamma^{*}$ dual to $\Gamma: \mathcal{S}$ has one three-cell for each face of $\Gamma^{*}$ (that is, for each vertex of $\Gamma$ ), one two-face for each face of $\Gamma^{*}$ and one for each edge of $\Gamma^{*}$ and one edge for each edge of $\Gamma^{*}$ and one for each vertex of $\Gamma^{*}$. Its vertices are the vertices of $\Gamma^{*}$ plus one, which will be called here the "central" vertex of $\mathcal{S}$.

With a hyperideal circle pattern is naturally associated a noncomplete hyperbolic metric on $\mathcal{S}$, which might have conical singularities along the edges of $\mathcal{S}$ corresponding to the vertices of $\Gamma^{*}$. This metric is obtained by giving to each three-cell of $\mathcal{S}$ the hyperbolic metric on a hyperideal polyhedron, such that the central vertex is either ideal—when considering Euclidean circle patterns — or strictly hyperidealwhen considering hyperbolic circle patterns. It is such that there is a well-defined notion of exterior dihedral angle at the edges of $\Gamma^{*}$ and this dihedral angle is equal to the intersection angle between the corresponding circles of the pattern.

The "hyperideal" circle pattern on the Euclidean (resp., hyperbolic) surface is then recovered by considering a family of horosphere centered at the "central" vertex (resp., the intersection with each three-cell of the hyperplane dual to the "central" vertex) and projecting on it, in the direction of the "central" vertex, the circle which is the boundary at infinity of the face opposite the "central" vertex. (This is explained in Sect. 4).

The construction works both ways: given a hyperideal circle pattern on a singular surface, one can recover a "polyhedral" object as described above. Moreover, the total angles around the singularities on the Euclidean (resp. hyperbolic) surface are equal to the total angles around the edges going to the "central" vertex, and the intersection angles between the circles are equal to the exterior dihedral angles at the edges which do not contain the "central" vertex. So proving Theorem 1.4 and Theorem 1.6 is equivalent to proving the existence and uniqueness of those polyhedral objects with given combinatorics, conical singularities along some edges, and dihedral angles.

\subsection{The Infinitesimal Rigidity}

A key idea of the proof - as in some of the references cited above on circle packings or "ideal" circle patterns-is to consider a wider range of hyperbolic metrics on the three-cells of $\mathcal{S}$, including some which do not allow isometric gluings of the cells or for which this gluing leads to "bad" singularities on the edges going to the central vertex. In the deformation method used here, however, it is used only in the proof of the infinitesimal rigidity of circle patterns.

The main point is that, among all those more general choices of hyperbolic metrics on the three-cells of $\mathcal{S}$, those which correspond to circle patterns are characterized as 
maxima of a function-which is simply the sum of the hyperbolic volumes of the three-cells - under some linear constraints on the dihedral angles. Since this function is strictly concave, the maxima are isolated, which proves the infinitesimal rigidity.

\subsection{The Results on Surfaces with Boundary}

All the results concerning surfaces with boundary-Theorems 1.10 and 1.11 -are consequences of Theorems 1.4 and 1.6 using a doubling argument to pass from a surface with boundary to a closed surface.

\section{Necessary Conditions}

\subsection{Elementary Properties of Circle Patterns}

It is the object of this section to prove that the conditions appearing in the statements of the main theorems given in the introduction are necessary. We first state some preliminary properties of some simple patterns of circles on a surface with at most one singularity, and the next subsection will show how similar arguments indicate that the conditions in the two theorems concerning circle patterns on closed surfaces are necessary.

Circles Intersecting at a Point The first statement describes a very simple situation, with a set of circles meeting at the only singular point of a Euclidean surface.

Lemma 3.1 Let $C_{E}(\kappa)$ (resp., $C_{H}(\kappa)$ ) be the complete Euclidean (resp., hyperbolic) metric on the plane with one conical singularity, with singular curvature $\kappa$ $(\kappa \in(-\infty, 2 \pi))$, and let $x_{0}$ be its singular point. Let $C_{1}, \ldots, C_{N}$ be a sequence of circles on $C_{E}(\kappa)$, containing $x_{0}$, such that the oriented tangents to the $C_{i}$ at $x_{0}$ appear in cyclic order. Let $\theta_{i}$ be the angle between $C_{i}$ and $C_{i+1}$ (with the convention that $\left.C_{N+1}=C_{1}\right)$. Then

$$
\sum_{i=1}^{N} \theta_{i}=2 \pi-\kappa
$$

Proof Let $c_{1}, \ldots, c_{N}$ be the centers of the $C_{i}$, as in Fig. 4. For $i=1, \ldots, N$, let $s_{i}$ be the oriented geodesic segment starting at $x_{0}$ and ending at $c_{i}$. Then the oriented angle at $x_{0}$ between $C_{i}$ and $s_{i}$ is equal to $\pi / 2$, so that the oriented angle between $s_{i}$ and $s_{i+1}$ is equal to $\theta_{i}$. So $\sum_{i=1}^{N} \theta_{i}$ is equal to the total angle at the conical point $x_{0}$, which is equal to $2 \pi-\kappa$.

\subsection{Necessary Conditions}

The Conditions of Theorem 1.4 Are Necessary We now check that the conditions appearing in Theorem 1.4 are necessary. Condition (1) is a restatement of the GaussBonnet theorem. We will see that condition (2) follows from arguments similar to 
Fig. 4 Lemma 3.1

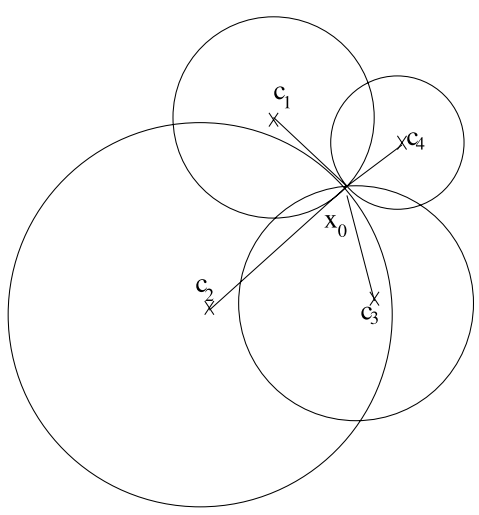

Fig. 5 Chains of intersecting circles

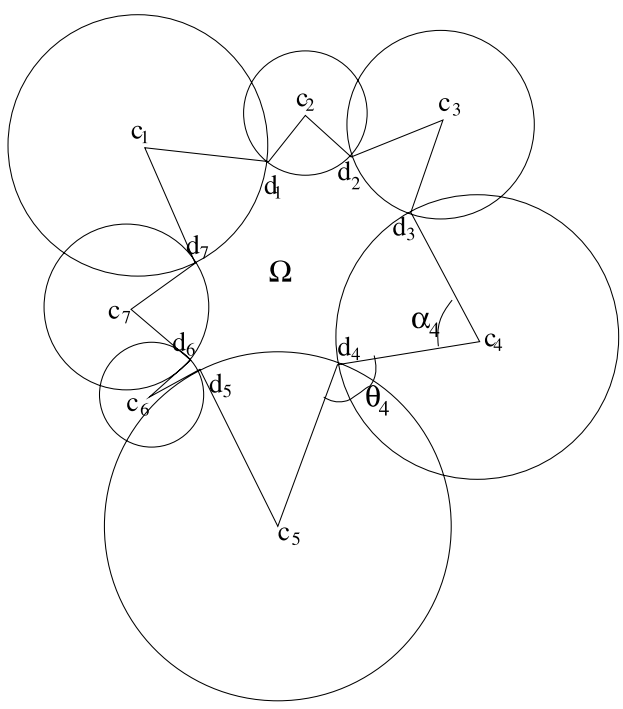

those used in the proof of Lemma 3.1, with the equality case a direct consequence of that statement.

Suppose that $\Gamma$ is the incidence graph of a circle pattern on a surface $\Sigma$ with a flat metric with conical singularities, as in Theorem 1.4, and let $\Omega$ be an admissible domain in $(\Sigma, \Gamma)$. Suppose first that $\partial \Omega$ is contained in the one-skeleton of $\Gamma$, so that $\partial \Omega$ is a disjoint union of closed curves $S_{1}, \ldots, S_{k}$ and, for each $i \in\{1, \ldots, k\}$, $S_{i}$ is made of a sequence of edges $\gamma_{1}^{i}, \ldots, \gamma_{N_{i}}^{i}$ of $\Gamma$. To each $S_{i}$ is also associated a sequence of circles, $C_{1}^{i}, \ldots, C_{N_{i}}^{i}$, such that $C_{j}^{i}$ intersects $C_{j+1}^{i}$ (including $j=N_{i}$, then $C_{N_{i}}^{i}$ intersects $C_{1}^{i}$ ), with $\gamma_{j}^{i}$ having as endpoints the vertices corresponding to $C_{j}^{i}$ and $C_{j+1}^{i}$. We consider $\gamma_{j}^{i}$ as realized as a geodesic segment with endpoints the centers $c_{j}^{i}$ and $c_{j+1}^{i}$ of $C_{j}^{i}$ and $C_{j+1}^{i}$, respectively. 
For each $j=1, \ldots, N$, let $d_{j}^{i}$ be the intersection point between $C_{j}^{i}$ and $C_{j+1}^{i}$ which is on the same side of $\gamma_{1}^{i} \cup \cdots \cup \gamma_{N_{i}}^{i}$ as $\Omega$. We call $\theta_{j}^{i}$ the angle at $d_{j}^{i}$ between $\left[d_{j}^{i}, c_{j}^{i}\right]$ and $\left[d_{j}^{i}, c_{j+1}^{i}\right]$, and $\alpha_{j}^{i}$ the angle at $c_{j}^{i}$ between $\left[c_{j}^{i}, d_{j-1}^{i}\right]$ and $\left[c_{j}^{i}, d_{j}^{i}\right]$, see Fig. 5.

Let $K$ be the sum of the singular curvatures of the metric in the domain bounded by the polygonal curves just described, i.e. $K$ is the sum of the singular curvatures attached by the function $\kappa$ to the faces of $\Gamma$ which are contained in $\Omega$. The GaussBonnet theorem, applied to the polygonal curve made of the segments $\left[c_{j}^{i}, d_{j}^{i}\right]$ and $\left[d_{j}^{i}, c_{j+1}^{i}\right]$, yields

$$
\sum_{i=1}^{k} \sum_{j=1}^{N_{i}}\left(\theta_{j}^{i}-\pi\right)+\left(\pi-\alpha_{j}^{i}\right)=2 \pi \chi(\Omega)-K
$$

so that

$$
\sum_{i=1}^{k} \sum_{j=1}^{N_{i}} \theta_{j}^{i}=2 \pi \chi(\Omega)-K+\sum_{i=1}^{k} \sum_{j=1}^{N_{i}} \alpha_{j}^{i},
$$

and the result follows.

Suppose now that the equality is attained in condition (2) of Theorem 1.4. It follows quite directly from the argument given earlier that, for each polygonal curve $S_{i}$ corresponding to one of the boundary component of $\Omega$, all the angles $\alpha_{j}^{i}$ are zero, so that the situation is exactly the one described in Lemma 3.1-in particular $\Omega$ is a face of $\Gamma$, as claimed.

Consider now the more general case where $\Omega$ is an admissible domain with boundary containing, in addition to edges of $\Gamma, m$ curves which are contained in faces of $\Gamma$. The same procedure can then be applied, associating a circle to each vertex of $\partial \Omega$. In addition we also associate a circle to each segment of $\partial \Omega$ which is contained in a face $f$ of $\Gamma$ : namely, the dual circle corresponding to $f$. This circle intersects orthogonally the circles corresponding to the two vertices of $\partial \Omega$ which are adjacent to it. Therefore, following the proof given above yields the same formula, except that to each segment of $\partial \Omega$ which is contained in a face of $\Gamma$ correspond two angles equal to $\pi / 2$ in (4), so that this left-hand side is increased by $m(\Omega) \pi$. This proves that the conditions in Theorem 1.4 are necessary.

Hyperbolic Surfaces The arguments showing that the conditions of Theorem 1.6 and of Theorem 1.11 are necessary then proceed exactly as in the proof of the corresponding Euclidean statements. Condition (1) is a consequence of the Gauss-Bonnet theorem, since the area of the surfaces appears in the formula. The fact that the second condition is also necessary can also be proved as in the Euclidean case, with only one difference, namely that the area of the domain which is considered-bounded by a disjoint union of polygonal curves-also comes in the Gauss-Bonnet formula, but with a sign which does not disturb the proof. 


\section{Some Hyperbolic Geometry}

A Short Introduction As already outlined in Sect. 2, it will be useful to consider some three-dimensional hyperbolic geometric objects, of a polyhedral nature, associated with the circle patterns on which our attention is focused. Those are obtained by gluing a hyperideal pyramid for each face of the graph $\Gamma^{*}$ dual to $\Gamma$, with a vertex "outside" $\Gamma^{*}$ which is either ideal—when considering Euclidean circle patterns-or strictly hyperideal—when considering hyperbolic circle patterns.

It is first necessary to recall the definitions of hyperideal polyhedra, and a basic result of Bao and Bonahon on their possible dihedral angles, and then some further (rather elementary) definitions concerning their volume and edge lengths. The next subsection recalls the Schläfli formula for hyperideal polyhedra, and the concavity property for their volume. The third and last subsection describes precisely the relation between a hyperideal circle pattern and the underlying three-dimensional cell complex with its hyperbolic metric, as well as the more general geometric objects which will appear in the proof of the infinitesimal rigidity of circle patterns.

\subsection{Ideal and Hyperideal Polyhedra}

Hyperideal Polyhedra When considering hyperideal polyhedra, it is helpful to make use of the Klein, or projective, model of $H^{3}$; it is a map from $H^{3}$ to the unit ball in Euclidean three-space, which sends hyperbolic geodesics to geodesic segments (see e.g. [16]). Compact hyperbolic polyhedra correspond in this model to polyhedra in the open unit ball, while ideal polyhedra correspond to polyhedra in the unit ball with all their vertices on the unit sphere, and hyperideal polyhedra correspond to polyhedra with all their vertices outside the open unit ball, but with all their edges intersecting this ball. A vertex of a hyperideal polyhedron is ideal if it sits on the unit sphere, strictly hyperideal otherwise. A hyperideal polyhedron is strictly hyperideal if all its vertices are strictly hyperideal.

To each edge of a compact/ideal/hyperideal polyhedron, we can associate its dihedral angle, which is the angle between the two faces of the polyhedron which meet at that edge. We will always consider here the exterior dihedral angle, which is defined as $\pi$ minus the angle measured in the interior of the polyhedron.

The possible exterior dihedral angles of hyperideal hyperbolic polyhedra have been described recently by Bao and Bonahon, extending previous results of Andreev [2] and Rivin [31] concerning ideal polyhedra. It can also be obtained as a consequence of (an extension of) a result of Rivin and Hodgson [29, 33] on compact hyperbolic polyhedra, see [34]. The statement uses the notion of admissible open path in a graph, which is defined as a simple path $\gamma$, beginning and ending at vertices of a face $f$, and not contained in the boundary of $f$.

Theorem 4.1 (Bao, Bonahon [3]) Let $\Gamma$ be the one-skeleton of a polytopal cellular decomposition of $S^{2}$. Let $w: \Gamma_{1} \rightarrow(0, \pi)$. There exists a hyperideal polyhedron with combinatorics dual to $\Gamma$ and (exterior) dihedral angles given by $w$ if and only if:

- for each simple closed curve $\gamma$ in $\Gamma$, the sum of the values of $w$ on the edges of $\gamma$ is at least $2 \pi$, with strict inequality unless $\gamma$ bounds a face, and 
- for each admissible open path $\gamma$ in $\Gamma$, the sum of the values of $w$ over the edges of $\gamma$ is strictly larger than $\pi$.

This hyperideal polyhedron is then unique (up to global hyperbolic isometries).

The equality case in the first condition corresponds exactly to the faces of $\Gamma$ corresponding to ideal vertices of the polyhedron.

Edge Lengths of Ideal and Hyperideal Polyhedra There is a natural projectively defined duality between points which are outside the closed unit ball in $\mathbb{R}^{3}$ and planes intersecting the unit ball (which are themselves identified with hyperbolic planes by the projective model of $H^{3}$ ). It is related to a duality between the hyperbolic and the de Sitter space, see e.g. [14, 18, 29, 33, 36, 44], but this aspect will not be used here. It associates to each point $x$ outside the unit ball the plane $p$ containing the intersection with the unit sphere of the cone with vertex at $x$ which is tangent to this unit sphere. It has a striking property: the intersection of $p$ with the unit ball, considered as a hyperbolic plane, is orthogonal to the intersections with the unit ball of all lines going through $x$, also considered as hyperbolic geodesics. The intersection of $p$ with the unit ball, considered as a hyperbolic plane, is called the hyperbolic plane dual to $x$.

Note that, given a hyperideal polyhedron, the hyperbolic planes dual to the strictly hyperideal vertices are disjoint. This is a direct consequence, using the projective definition of the duality, of the fact that the line going through two vertices always intersects the unit ball in $\mathbb{R}^{3}$.

Given a hyperideal hyperbolic polyhedron $P$, the associated truncated polyhedron $P_{t}$ (defined in [3]) is obtained by cutting off each strictly hyperideal vertex by its dual plane. Its vertices are "usual" points of $H^{3}$, except for the ideal vertices of $P$, which remain vertices of $P_{t}$. The faces of $P_{t}$ are either truncated faces of $P$, or "new" faces, which are orthogonal to all adjacent faces.

Given an edge of $P$ which has as endpoints two strictly hyperideal vertices, its length is defined as the length of the corresponding edge of $P_{t}$. To define the lengths of the edges with one or two ideal vertices as their endpoints, it is necessary to choose, for each ideal vertex $v$ of $P$, a horosphere $h$ "centered" at $v$; one then defines the length of an edge of $P$, with endpoints $v_{1}$ and $v_{2}$ as the oriented distance between the plane dual to $v_{1}$ (if $v_{1}$ is strictly hyperideal) or the horosphere chosen for $v_{1}$ (if $v_{1}$ is ideal) to the plane dual to $v_{2}$ or the horosphere chosen for $v_{2}$. Replacing a horosphere centered at a vertex $v$ by another one clearly adds a constant - the oriented distance between the two horospheres- to all the lengths of the edges with endpoint $v$, so that, if $P$ has $n$ ideal vertices, the lengths of the edges of $P$ are defined up to the addition of $n$ constants, one for each ideal vertex (the constants being added to the lengths of the edges containing the respective vertex).

Obviously, what has been said of hyperideal polyhedra is also true of hyperideal triangles, which are defined in the same way using the projective model of $H^{2}$. This leads to the following elementary proposition.

Proposition 4.2 Two hyperideal triangles are isometric if and only if they have the same number of ideal vertices and the same edge lengths. Any nontrivial first-order 


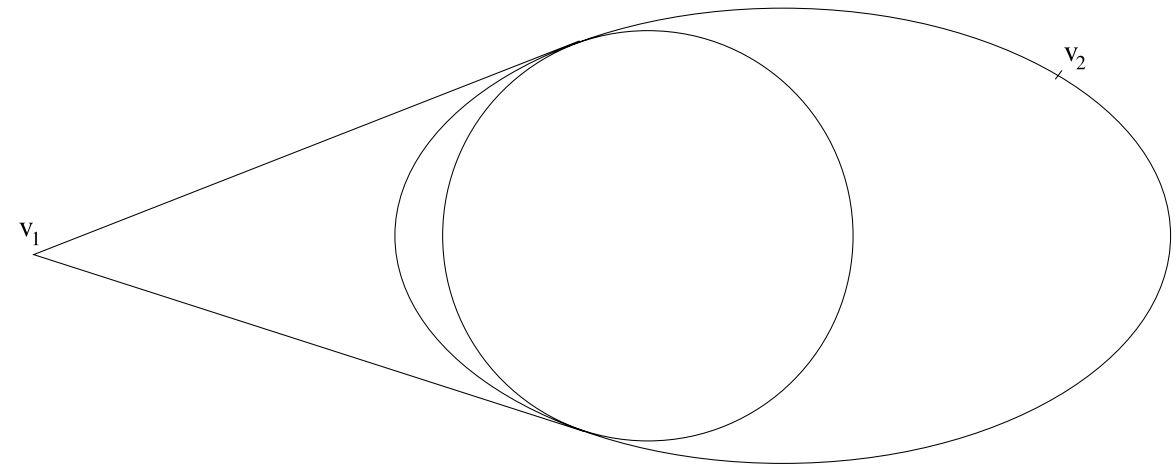

Fig. 6 Possible positions of $v_{2}$ given $v_{1}$ and the edge length

deformation of a hyperideal triangles induces a nonzero first-order variation of its edge lengths.

Proof Another formulation of the first statement is that hyperideal triangles are uniquely determined, up to global isometries, by their edge lengths. Consider first a strictly hyperideal triangle (i.e. with no ideal vertex). Using the projective model of $H^{2}$, one can choose arbitrarily the position of the first vertex, $v_{1}$; the fact that the edge going from $v_{1}$ to $v_{2}$ has given length is equivalent, in the projective model, to the fact that $v_{2}$ is on a segment of an ellipse tangent to the unit circle, with endpoints at the tangency points, as depicted in Fig. 6. Different choices of points on that segment are equivalent up to an isometry.

Then, given the position of $v_{1}$ and $v_{2}$, the position of $v_{3}$ is uniquely determined (up to an isometry) by the condition that it is contained in two ellipses, one determined by the length of the edge between $v_{1}$ and $v_{3}$, the other by the length of the edge from $v_{2}$ to $v_{3}$.

The same argument can be used if $v_{1}$ is an ideal vertex, using the fact that, given $v_{1}$ and a horosphere $h_{1}$ "centered" at $v_{1}$, the possible positions of $v_{2}$ such that the length between $v_{1}$ and $v_{2}$ (measured with respect to the choice of $h_{1}$ ) is given, are on a circle tangent to the unit circle at $v_{1}$. The same argument works when $v_{1}$ and $v_{2}$ are ideal points, then, given the choice of $v_{1}$ and $h_{1}$, the position of $h_{2}$ can be chosen arbitrarily on the unit circle but determines the horosphere $h_{2}$ "centered" at $v_{2}$. Finally, if all three vertices are ideal, the statement is trivial, since all ideal triangles are isometric, and there is only one possible set of edge lengths (up to the addition of three arbitrary constants, corresponding to the three ideal vertices).

The proof of the second part of the statement can be done exactly along the same lines, by considering a first-order variation of the edge lengths and the implied conditions on the first-order displacement of the vertices.

\subsection{The Volume of Hyperideal Polyhedra}

It follows from the definition of the truncated polyhedron $P_{t}$ associated to a hyperideal polyhedron $P$ that $P_{t}$ always has finite volume, leading us to define the volume 
of $P$ as the volume of $P_{t}$ (of course, the intersection of hyperbolic three-space with the interior of $P$, in the projective model of $H^{3}$, does not have finite volume unless all vertices of $P$ are ideal).

The Schläfli Formula Consider first a compact polyhedron $P$. The classical Schläfli formula (see e.g. [26]) gives the first-order variation of the volume of $P$ under a first-order deformation

$$
d V=\frac{1}{2} \sum_{e} l_{e} d \theta_{e},
$$

where the sum is over the edges of $P, l_{e}$ is the length of edge $e$, and $\theta_{e}$ is its (exterior) dihedral angle. Note that no minus sign is needed in the formula because we consider the exterior dihedral angles.

This formula remains valid for polyhedra having some ideal vertices (see [26, 31]) if one uses the definition of the edge lengths given earlier, defined up to the addition of one constant for each ideal vertex, and under the condition that the ideal vertices remain ideal in the first-order deformation considered. Note that formula (5) does not depend on the choice of the horospheres at the vertices, because the sum of the exterior dihedral angles of the edges containing an ideal vertex is always equal to $2 \pi$ (this is seen by considering the link of the ideal vertex, which is an Euclidean polygon with edge lengths equal to those exterior dihedral angles).

Since both the volume and the edge lengths of a hyperideal polyhedron are defined by reference to the associated truncated polyhedron, it follows that (5) also holds for hyperideal polyhedra.

A Technical Lemma on Convex Functions Before we proceed, we need the following elementary statement on properties of concave function. It is taken from [39], and we leave the proof, which is simple, to the reader.

Remark 4.3 Let $\Omega \in \mathbb{R}^{N}$ be a convex subset, and let $f: \Omega \rightarrow \mathbb{R}$ be a smooth, strictly concave function. Let $\rho: \mathbb{R}^{N} \rightarrow \mathbb{R}^{p}$ be a linear map, with $p<N$, and let $\bar{\Omega}:=\rho(\Omega)$. Define a function

$$
\begin{aligned}
\bar{f}: \bar{\Omega} & \rightarrow \\
y & \mapsto \max _{x \in \rho^{-1}(y)} f(x) .
\end{aligned}
$$

Then $\bar{\Omega}$ is convex, and $\bar{f}$ is a smooth, strictly concave function on $\bar{\Omega}$.

A Key Convexity Property The key point of this section is the fact, already pointed out in [39] and used in [41], that the volume of hyperideal polyhedra is a strictly concave function of the dihedral angle. We give here a very short outline of the proof for the sake of the curious reader, but refer to [39] for a complete proof. The ideas used here are close to those in [9, 31, 39].

Lemma 4.4 Let $\Gamma$ be the one-skeleton of a (polytopal) cellular decomposition of the sphere, and let $V \subset \Gamma_{2}$ be a subset of the set of its faces. Let $P_{\Gamma}$ be the space of hyperideal polyhedra with combinatorics dual to $\Gamma$, such that the ideal vertices 
correspond exactly to the faces of $\Gamma$ which are in $V$. The volume, considered as a function on $P_{\Gamma}$, is a strictly concave function of the dihedral angles.

Main ideas of the proof We consider here only the case of strictly hyperideal polyhedra, otherwise the proof is similar but slightly more complicated, as should be clear from Sect. 5. Recall that, by Theorem 4.1, those hyperideal polyhedra are parameterized by their possible dihedral angles.

It is necessary to consider first the simplest case, the hyperideal simplices. One can prove, along the ideas of the (direct) proof of Proposition 4.2, that hyperideal simplices are rigid: any nontrivial first-order deformation of a hyperideal simplex induces a nonzero first-order variation of its edge lengths.

Using the Schläfli formula, this implies that the Hessian of the volume function with respect to the dihedral angles — which by (5) is the matrix of the derivatives of the edge lengths with respect to the dihedral angles-is nondegenerate, since a nonzero vector in its kernel would precisely correspond to a first-order variation of the dihedral angles inducing no first-order variation of the edge lengths.

Therefore, the signature of the Hessian of the volume (with respect to the dihedral angles) is constant over the space of hyperideal simplices (which is connected by Theorem 4.1). To prove that the volume is a strictly concave function of the dihedral angles, it is therefore sufficient to check, by an explicit computation, that it is true for a well chosen simplex (for instance one with maximal symmetry), which is done in the appendix of [39].

We now consider a hyperideal polyhedron $P$. We choose a vertex $v$ of $P$, and add edges to the faces of $P$ so as to subsdivide all faces of $P$ into triangles, with all faces containing $P$ subdivided by adding only edges containing $v$. This yields a new polyhedron $P^{\prime}$, with the same edges as $P$ plus additional edges where the exterior dihedral angle is 0 . Then we define a triangulation of the interior of $P^{\prime}$ into simplices, by simplices with one vertex at $v$ and three vertices which are the vertices of a face of $P^{\prime}$ which does not contain $v$. Let $S_{1}, \ldots, S_{N}$ be the simplices in this decomposition. Then the $S_{i}$ are the cells of a natural cell complex, with two kinds of edges: the "exterior" edges, which are either edges of $P$ or contained in faces of $P$, and the "interior" edges, which are in the interior of $P$ and have $v$ as one of their endpoints.

For each $i=1, \ldots, N$, let $\mathcal{A}_{i}$ be the space of possible interior dihedral angles defined on $S_{i}$ by identifying it to a strictly hyperideal simplex. Then let $\mathcal{A}:=\prod_{i=1}^{N} \mathcal{A}_{i}$. There is a natural function $\mathcal{V}: \mathcal{A} \rightarrow \mathbb{R}$, sending an element $a \in \mathcal{A}$ to the sum of the volumes assigned to the $S_{i}$ by $a$.

Each point $a \in \mathcal{A}$ determines an "extended" hyperbolic metric corresponding to a hyperideal simplex on each of the $S_{i}, i=1, \ldots, N$, but in general not an "extended" hyperbolic metric corresponding to a hyperideal polyhedron on $P$, because:

- It is in general not possible to glue the $S_{i}$ isometrically along their faces.

- Even if this gluing is possible, the resulting hyperbolic metric might have nontrivial holonomy along the "interior" edges: the total angle around those edges could be different from $2 \pi$.

Actually it is not difficult to check that those conditions are sufficient, so that, if they are realized, then $a$ determines an identification of $P$ with a hyperideal polyhedron, 
which is isometric on each of the $S_{i}, i=1, \ldots, N$. There is an element $a_{0} \in \mathcal{A}$ associated to the "original" situation of $P$, since it determines an "extended" hyperbolic metric on each of the $S_{i}, i=1, \ldots, N$.

Let $E^{\prime}$ be the set of edges of $P^{\prime}$, and let $E_{i}$ be the set of "interior" edges of the cellular decomposition of $P^{\prime}$ as the union of the $S_{i}, i=1, \ldots, N$. Let $\mathcal{W}$ be the vector space of maps $w: E^{\prime} \cup E_{i} \mapsto \mathbb{R}$. There is a natural affine map $\Phi: \mathcal{A} \mapsto \mathcal{W}$, sending an element $a \in \mathcal{A}$-i.e. an assignment of dihedral angles to each of the edges of the $S_{i}, i=1, \ldots, N-$ to a map $w(a): E^{\prime} \cup E_{i} \mapsto \mathbb{R}$, defined by:

- For each $e \in E^{\prime}, w(a)(e)$ is $\pi$ minus the sum of the values of $a$ on the edge $e$ for all the $S_{i}$ which contain $e$.

- For each $e_{i} \in E_{i}, w(a)\left(e_{i}\right)$ is $2 \pi$ minus the sum of the values of $a$ on the edge $e$ for all the $S_{j}$ which contain $e_{i}$.

For instance, $w\left(a_{0}\right)$ is zero on all interior edges, and is equal to the (exterior) dihedral angle of $P^{\prime}$ on all edges which are edges of $P^{\prime}$.

Let $a \in \mathcal{A}$, remark that the lengths of each edge are the same for all the $S_{i}$ containing it if and only if $a$ is a critical point of $\mathcal{V}$ on $w^{-1}(w(a))$. This is a direct consequence of the Schläfli formula (5). Indeed, if the lengths assigned to each edge are the same for all simplices containing it, then it is quite clear that any first-order variation of $a$ which does not change the sum of the angles at each edge leaves $\mathcal{V}$ unchanged (at first order). Conversely, if two simplices, say $S_{i}$ and $S_{j}$, both contain an edge $e \in E^{\prime} \cup E_{i}$ and $a$ assigns different lengths to $e$ as an edge of $S_{i}$ and of $S_{j}$, then there is a first-order deformation of $a$, tangent to $w^{-1}(w(a))$, which increases the angle of $e$ in $S_{i}$ and decreases the edge length of $e$ in $S_{j}$ by the same amount, so that $\mathcal{V}$ varies.

Since $w$ is an affine function, $\mathcal{A}$ is foliated by the level sets of $w$, which are affine submanifolds. But $\mathcal{V}$ is a sum of strictly concave functions, so it is strictly concave. It follows that, in a neighborhood of $a_{0}$ - which is a local maximum of $\mathcal{V}_{\mid w^{-1}\left(w\left(a_{0}\right)\right)}$ each level set of $w$ contains a unique local maximum of the restriction of $\mathcal{V}$, so that each small variation of the dihedral angles of $P^{\prime}$ (resp., $P$ ) is obtained uniquely by a small deformation of $P^{\prime}$ (resp., $P$ ). So Remark 4.3 shows that the volume of a strictly concave function on hyperideal polyhedra in the neighborhood of $P$.

This argument is very close to the one that is used in Sect. 5 to prove the infinitesimal rigidity of hyperideal circle patterns, and can be used as a "toy model" for it. The proof in Sect. 5 is a little more complicated since it has to take into account the possibility of ideal vertices.

\subsection{The Three-Dimensional Cone-Manifold Associated to a Circle Pattern}

Euclidean Circle Patterns Let $\Gamma$ be a graph embedded in a closed surface $\Sigma$, as in the setting of Theorem 1.4. Following the outline given in Sect. 2, we define a cell complex $\mathcal{S}_{\Sigma, \Gamma}$ associated to $\Gamma$ as the cone over $\Gamma^{*}$. It has:

- One vertex for each vertex of $\Gamma^{*}$, and an additional one, $v_{0}$, common to all the three-cells.

- One three-cell for each face $f$ of $\Gamma^{*}$. If $f$ has $p$ vertices, then the corresponding three-cell of $\mathcal{S}_{\Sigma, \Gamma}$ has $p+1$ vertices, corresponding to the vertices of $f$ and to the "central" vertex, $v_{0}$, which is common to all three-cells. 
Fig. 7 Proposition 4.5

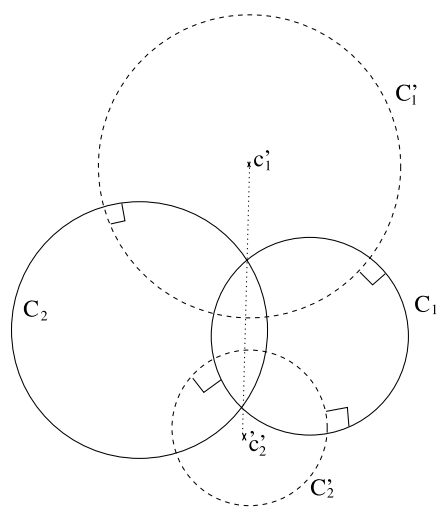

- One two-face for each face $f$ of $\Gamma^{*}$-corresponding to the face of the three-cell associated to $f$ which is opposite to $v_{0}$-and one for each edge of $\Gamma^{*}$. We call "horizontal" the faces of the first kind, and "vertical" the others.

- One edge for each edge of $\Gamma^{*}$, and one for each vertex of $\Gamma^{*}$. Again we call "horizontal" the edges of the first type, and "vertical" the others.

We call $S_{1}, \ldots, S_{N}$ the three-cells of $\mathcal{S}_{\Sigma, \Gamma}$.

The definition of $\mathcal{S}_{\Sigma, \Gamma}$ up to this point is entirely topological. However, it is also desirable to put on this cell complex a hyperbolic metric, or more precisely the structure of a (noncompact) hyperbolic cone-manifold, with cone singularities along the vertical edges, and with a boundary composed of parts of the horizontal faces and of the horizontal edges. To this end, it will be useful to consider two simple propositions. The first is an elementary statement of plane geometry, which will clear up the following considerations. We leave the proof of this very classical proposition to the reader, who might also be convinced by Fig. 7 .

Proposition 4.5 Let $C_{1}, C_{2}$ be two circles in the Euclidean (resp., hyperbolic) plane which intersect in two points. Let $C_{1}^{\prime}, C_{2}^{\prime}$ be two nonintersecting circles which are both orthogonal to both $C_{1}$ and $C_{2}$. Then the intersection points of $C_{1}$ and $C_{2}$ lie on the line containing the centers of $C_{1}^{\prime}$ and $C_{2}^{\prime}$.

The next proposition describes a hyperbolic polyhedron associated to a simple pattern of circles in the Euclidean plane. It will serve in the following as a "building block" for the construction of a hyperbolic cone-manifold structure associated to a hyperideal circle pattern. A similar statement holds in the hyperbolic case, but it is kept separate so as to avoid complicated notation.

Consider the following situation. $C_{0}$ is an oriented circle in the Euclidean plane, and $C_{1}^{\prime}, \ldots, C_{N}^{\prime}$ are oriented circles bounding disjoint disks, orthogonal to $C_{0}$, appearing in cyclic order along it, with $C_{i}^{\prime}$ having center $c_{i}^{\prime}$ and radius $r_{i}, i=1, \ldots, N$, see Fig. 9. Let $d_{i}$ be the Euclidean distance between $c_{i}^{\prime}$ and $c_{i+1}^{\prime}$. The Euclidean plane is identified, as above, with a horosphere $H_{0}$ "centered" at a point $v_{0} \in \partial_{\infty} H^{3}$, and we consider the hyperbolic Gauss map $G: H_{0} \rightarrow \partial_{\infty} H^{3}$.

We now construct a polyhedron $P$ associated to $C_{0}$ and to the circles $C_{i}^{\prime}$, using the projective model of $H^{3}$, with one vertex at $v_{0}$ and the other at the hyperideal 
Fig. 8 Proposition 4.6

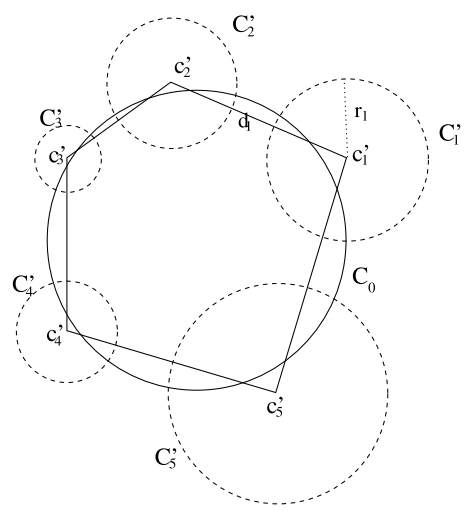

points $v_{1}^{\prime}, \ldots, v_{N}^{\prime}$ which are the points dual to the hyperbolic planes with boundary at infinity $G\left(C_{1}^{\prime}\right), \ldots, G\left(C_{N}^{\prime}\right)$. Note that the construction of $P$ uses the projective model of $H^{3}$, but the resulting polyhedron-considered as a hyperideal polyhedron with one ideal vertex - does not depend on any choice once $C_{0}$ and the $C_{i}^{\prime}$ (considered as circles in the Euclidean plane) are fixed.

Proposition 4.6 $P$ is a hyperideal polyhedron, with ideal vertex $v_{0}$ and strictly hyperideal vertices at $v_{1}^{\prime}, \ldots, v_{N}^{\prime}$, it is combinatorially a pyramid with vertex $v_{0}$. Moreover, defining the edge lengths involving $v_{0}$ with respect to the horosphere $H_{0}$ :

1. The length of the edge $\left[v_{0}, v_{i}^{\prime}\right]$ is $-\log \left(r_{i}\right)$.

2. The length of the edge $\left[v_{i}^{\prime}, v_{i+1}^{\prime}\right]$ depends only on $d_{i}, r_{i}$ and $r_{i+1}$.

3. The (exterior) dihedral angle at the edge $\left[v_{i}^{\prime}, v_{i+1}^{\prime}\right]$ is equal to the angle between $\left[c_{i}^{\prime}, c_{i+1}^{\prime}\right]$ and $C_{0}$, measured in the interior of the intersection of the interior of $C_{0}$ with the interior of the polygon $p$ with vertices the $c_{i}^{\prime}, i=1, \ldots, N$.

4. The (exterior) dihedral angle at the edge $\left[v_{0}, v_{i}^{\prime}\right]$ is equal to the exterior angle of $p$ at $c_{i}^{\prime}$.

Point (2) could be made more explicit at the cost of a fairly classical computation, but a precise expression will not be necessary here.

Proof It is simplest to consider the first point in the Poincaré half-space model, taking as $H_{0}$ the plane $\{z=1\}$. Then the Gauss map is simply the vertical projection on the plane $\{z=0\}$, which corresponds to $\partial_{\infty} H^{3} \backslash\left\{v_{0}\right\}$. So the hyperbolic plane with boundary at infinity $G\left(C_{i}^{\prime}\right)$ corresponds to a half-sphere of radius $r_{i}$, and its hyperbolic distance to $H_{0}$ is obtained by integrating $d z / z$ from $r_{i}$ to 1 , so it is equal to $-\log \left(r_{i}\right)$. This, along with the definition of the length of the edges of hyperideal polyhedra, proves the first point.

The second point can be proved in the same manner; still in the Poincaré half-space model, the hyperbolic planes with boundary at infinity $G\left(C_{i}^{\prime}\right)$ and $G\left(C_{i+1}^{\prime}\right)$ correspond to half-spheres of radii $r_{i}$ and $r_{i+1}$, respectively, and with centers at Euclidean distance $d_{i}$ on the plane $\{z=0\}$. Computing the distance between them is thus a simple exercise (which we leave to the reader) and the result is a function only of $r_{i}, r_{i+1}$ and $d_{i}$. 
The third and last points can be proved using the same model. In the Poincare halfspace model, the faces of $P$ correspond to the half-sphere with boundary the circle $G\left(C_{0}\right)$, which is contained in the plane $\{z=0\}$, and the vertical strips intersecting the plane $\{z=0\}$ at the edges of the polygon $p$ with vertices the images by $G$ of the $c_{i}^{\prime}$. By the conformality of the Poincaré half-space model, the hyperbolic angles between those faces are the same as the Euclidean angles, and the result follows directly.

Remark 4.7 Suppose that some of the $C_{i}^{\prime}$ are replaced by points, so that some of the vertices of $P$ other than $v_{0}$ are ideal vertices. Choose for each of those ideal vertices the horosphere which is tangent to $H_{0}$. Then points (2), (3) and (4) of the previous proposition still hold. Point (1) holds as well when $v_{i}^{\prime}$ is strictly hyperideal, while the length of the edge going from $v_{0}$ to $v_{i}^{\prime}$ is zero when $v_{i}^{\prime}$ is ideal.

The proof of this remark uses exactly the same arguments as the proof of the previous proposition. Proposition 4.6 and this remark, along with Proposition 4.2, make it possible to define a hyperbolic metric associated to a hyperideal circle pattern.

Definition 4.8 Let $C$ be a hyperideal circle pattern embedded in $\Sigma$, with incidence graph $\Gamma$. We call $H(C)$ the noncomplete metric defined on the complement of the vertical edges of $\mathcal{S}$, defined from $C$ by gluing one hyperideal hyperbolic pyramid for each face $f$ of $\Gamma^{*}$, as described in Proposition 4.6, taking as $C_{0}$ the circle corresponding to $f$ (or more precisely to the dual vertex of $\Gamma$ ) and as $C_{1}^{\prime}, \ldots, C_{N}^{\prime}$ the dual circles corresponding to the vertices of $f$ (or more precisely to the dual faces of $\Gamma$ ). Those pyramids are glued isometrically along their "vertical" faces, which correspond to the edges of $\Gamma^{*}$.

Note that it is indeed possible to glue the pyramids along their vertical faces since, by Proposition 4.6, corresponding faces in pyramids corresponding to adjacent faces of $\Gamma^{*}$ are triangles with the same edge lengths, so that, by Proposition 4.2, they are isometric.

Proposition 4.9 The completion of $H(C)$ is a hyperbolic metric on $\mathcal{S}$ with conical singularities along the "vertical edges". For each such edge e, corresponding to a vertex $v$ of $\Gamma^{*}$ (that is, to a face $v^{*}$ of $\Gamma$ ) the total angle around $e$ is equal to $2 \pi$ minus the singular curvature $\kappa\left(v^{*}\right)$ of $C$ at $v^{*}$.

Proof The completion of $H(C)$ is obtained by adding a line for each vertical edge of $\mathcal{S}$. The holonomy of $H(C)$ around each such edge is the composition of a translation along the edge and a rotation with axis equal to the edge. Proving that the completion is a metric with conical singularities is equivalent to proving that the translation component vanishes.

On each three-cell $S_{i}$ of $\mathcal{S}$, one can define a "Busemann function" $B_{S_{i}}$ corresponding to the point at infinity $v_{0}$, as the "oriented distance" to the horosphere $\mathrm{H}_{0}-0$ - that is, $B_{S_{i}}$ is equal to the distance to $H_{0}$ for points which are on the side of $H_{0}$ opposite to $x_{0}$, and to minus the distance to $H_{0}$ for points which are on the same side of $H_{0}$ as $x_{0}$. Proving that the translation component of the holonomy along a "vertical" edge $e$ vanishes is clearly equivalent to proving that the holonomy acts trivially on $B_{S_{i}}$, for 
each three-cell $S_{i}$ adjacent to $e$. But when one considers the three-cells adjacent to $e$, in cyclic order, the point of $e$ where the functions $B_{S_{i}}$ vanish remains the same-it is the intersection of $e$ with $H_{0}$. So, after completing a turn around $e$, the function $B_{S_{i}}$ remains the same, so that the translation component of the holonomy remains the same, and the completion of $H(C)$ is indeed a hyperbolic metric with conical singularities.

The total angle around a "vertical" edge $e$ of $\mathcal{S}$ is obtained by summing the interior dihedral angles at $e$ of the three-faces adjacent to it. Since those angles are equal to the angles at the vertex of $\Gamma^{*}$ corresponding to $e$ of the polygons corresponding to the faces of $\Gamma^{*}$, the interior dihedral angles at $e$ of the three-cells adjacent to it sum to the total angle around the singular point at the center of the circle corresponding to $v$, i.e. to $2 \pi-\kappa\left(v^{*}\right)$.

The metric which is constructed in this manner is not complete; in addition to the conical singularities, it has a boundary, which is "polyhedral", i.e. each of its points has a neighborhood in which the boundary looks either like a plane or like the neighborhood of an edge in a hyperbolic polyhedron.

A Property of Those Hyperbolic Metrics The hyperbolic metrics constructed on $\mathcal{S}$ above have one important property that is not quite apparent yet: it is possible to choose for each cell in $\mathcal{S}$ a horosphere, centered at the "central" vertex, so that the horospheres "match" along the vertical faces, as in the next definition.

Definition 4.10 Let $h$ be a hyperbolic metric on $\mathcal{S}$, with conical singularities along the vertical edges, which is obtained by gluing the hyperbolic metrics on the cells corresponding to the identification of each cell with a hyperideal polyhedron. We say that $h$ is consistent if it is possible to choose, for each cell $C_{i}$ of $\mathcal{S}$, a horosphere $H_{i}$ in $C_{i}$ centered at the "central" vertex, so that, if $C_{i}$ and $C_{j}$ share a two-dimensional face $f$, then $H_{i} \cap f=H_{j} \cap f$.

The way the metric $H(C)$ was constructed implies that:

Remark $4.11 H(C)$ is consistent.

Proof This follows directly from the construction of $H(C)$, which was made in each cell $C_{i}$ of $\mathcal{S}_{\Sigma, \Gamma}$ with respect to a horosphere $H_{0}$, which we can rename as $H_{i}$. Then the $H_{i}$ have precisely the requested property.

Conversely, any hyperbolic metric which is consistent is related to a hyperideal circle pattern.

Remark 4.12 Let $h$ be a hyperbolic metric on $\mathcal{S}_{\Sigma, \Gamma}$ with conical singularities on the vertical edges. Suppose that the restriction of $h$ to each three-dimensional cell of $\mathcal{S}_{\Sigma, \Gamma}$ is the metric induced on a hyperideal polyhedron, with an ideal vertex at $v_{0}$, and that $h$ is consistent. Suppose moreover that the boundary of $\mathcal{S}_{\Sigma, \Gamma}$ for $h$ is locally convex, i.e. the exterior dihedral angle is positive at each "horizontal" edge of $\mathcal{S}_{\Sigma, \Gamma}$. For each cell $C_{i}$ of $\mathcal{S}_{\Sigma, \Gamma}$, let $H_{i}$ be the part of horosphere appearing in 
Definition 4.10. Then the $H_{i}$ are isometric to Euclidean polygons which can be glued along their edges to obtain a Euclidean metric with conical singularities, which has a hyperideal circle pattern with incidence graph $\Gamma$ and intersection angles equal to the exterior dihedral angles between the "horizontal" faces of $\left(\mathcal{S}_{\Sigma, \Gamma}, h\right)$.

Proof The fact that the $H_{i}$ are isometric to Euclidean polygons follows from the elementary properties of horospheres. They can be glued isometrically along their edges because, if two cells $C_{i}$ and $C_{j}$ share a two-dimensional face $f$, then $H_{i} \cap f=$ $H_{j} \cap f$, so that those edges of $H_{i}$ and $H_{j}$ can be glued isometrically, to obtain an Euclidean metric with conical singularities.

Each of the $H_{i}$ is the orthogonal projection of the corresponding cell $C_{i}$, so that its vertices lie on an Euclidean circle $c_{i}$ - the projection of the boundary at infinity of the "horizontal" face of $C_{i}$. The convexity condition in the statement means precisely that the interior of $c_{i}$ intersects no vertical edge of $\mathcal{S}_{\Sigma, \Gamma}$, so that the $c_{i}$ indeed constitute a hyperideal circle pattern. It is then clear that the intersection angle between $c_{i}$ and $c_{j}$ is equal to the exterior dihedral angle between the "horizontal" faces of $C_{i}$ and $C_{j}$.

Hyperbolic Circle Patterns It is also possible to use the same construction for hyperideal circle patterns on a closed surface endowed with a singular hyperbolic metric. The main difference is that the "central" vertex $v_{0}$ is now strictly hyperideal rather than ideal. So the situation we consider is now as follows. $C_{0}$ is an oriented circle in the hyperbolic plane, and $C_{1}^{\prime}, \ldots, C_{N}^{\prime}$ are oriented circles bounding disjoint disks, orthogonal to $C_{0}$, appearing in cyclic order along it, with $C_{i}^{\prime}$ having center $c_{i}^{\prime}$ and radius $r_{i}, i=1, \ldots, N . d_{i}$ is the hyperbolic distance between $c_{i}^{\prime}$ and $c_{i+1}^{\prime}$. The hyperbolic plane is identified with a totally geodesic plane $P_{0}$ with dual point $v_{0}$, and we consider the hyperbolic Gauss map $G: H_{0} \rightarrow \partial_{\infty} H^{3}$. Let $v_{1}^{\prime}, \ldots, v_{N}^{\prime}$ be the points dual to the hyperbolic planes with boundary at infinity $G\left(C_{1}^{\prime}\right), \ldots, G\left(C_{N}^{\prime}\right)$, and let $P$ be the polyhedron with vertices $v_{0}, v_{1}^{\prime}, \ldots, v_{N}^{\prime}$. The analog of Proposition 4.6 is as follows.

Proposition 4.13 $P$ is a hyperideal polyhedron, with strictly hyperideal vertices at $v_{0}, v_{1}^{\prime}, \cdots, v_{N}^{\prime}$, it is combinatorially a pyramid with vertex $v_{0}$. Moreover:

1. the length of the edge $\left[v_{0}, v_{i}^{\prime}\right]$ depends only on $r_{i}$;

2. the length of the edge $\left[v_{i}^{\prime}, v_{i+1}^{\prime}\right]$ depends only on $d_{i}, r_{i}$ and $r_{i+1}$;

3. the (exterior) dihedral angle at the edge $\left[v_{i}^{\prime}, v_{i+1}^{\prime}\right]$ is equal to the angle between $\left[c_{i}^{\prime}, c_{i+1}^{\prime}\right]$ and $C_{0}$, measured in the interior of the intersection of the interior of $C_{0}$ with the interior of the polygon $p$ with vertices the $c_{i}^{\prime}, i=1, \ldots, N$; and

4. the (exterior) dihedral angle at the edge $\left[v_{0}, v_{i}^{\prime}\right]$ is equal to the exterior angle of $p$ at $c_{i}^{\prime}$.

Points (1) and (2) could be made more explicit at the cost of a fairly classical computation (which is even easy for point (1)), but a precise expression will not be necessary here.

Proof For the first point note that, by definition of the edge lengths of a hyperideal polyhedron, the length of the edge between $v_{0}$ and $v_{i}^{\prime}$ is equal to the distance be- 
tween $P_{0}$ and the hyperbolic plane with boundary at infinity $G\left(C_{i}^{\prime}\right)$. Clearly this only depends on the radius of the circle in $P_{0}$ which is the orthogonal projection of $C_{i}^{\prime}$.

The second point can be checked in the same manner, now the length is equal to the hyperbolic distance between the hyperbolic planes with boundaries at infinity $G\left(C_{i}^{\prime}\right)$ and $G\left(C_{i+1}^{\prime}\right)$, and again it only depends on the radii of $C_{i}^{\prime}$ and $C_{i+1}^{\prime}$ and on the distances between their centers.

Finally the third and last points can be proved as in the Euclidean setting.

It follows, as in the Euclidean case, that it is possible to glue isometrically hyperideal pyramids, corresponding to the faces of $\Gamma^{*}$, to obtain a hyperbolic conemanifold with polyhedral boundary canonically associated to a hyperideal circle pattern on a hyperbolic surface.

Conversely, it is possible to reconstruct a hyperideal circle pattern on a hyperbolic surface with conical singularities (at the centers of the dual circles) from such a gluing of hyperideal pyramids. This follows from the same argument as the one used in Remark 4.12, with the difference that the projection is now on the planes dual to $v_{0}$ in each three-cell of $\mathcal{S}(\Sigma, \Gamma)$.

\section{Infinitesimal Rigidity}

\subsection{Introduction and Outline}

This section contains the proof, in the different situations which are considered, of the infinitesimal rigidity of hyperideal circle patterns: any first-order deformation of such a pattern-including a deformation of the underlying singular Euclidean or hyperbolic metric - induces a first-order deformation of either the intersection angles between the circles or the singular curvatures at the cone singularities.

The main idea used here is not original-it originates in papers of Rivin [30] and Brägger [9] on ideal polyhedra, and was also related to or used in [4, 12, 23, 24, 32, 38]. Its extension to hyperideal polyhedra was already used to some extent in [39, 41].

The next subsection describes some relevant spaces of assignments of angles to the cells of $\mathcal{S}$, which are then used in the infinitesimal rigidity proof. The argument is slightly simpler when one considers hyperbolic circle patterns, so this case is considered first.

\subsection{Spaces of Angle Assignments}

To each graph $\Gamma$ on a closed surface, we have associated the cellular complex $\mathcal{S}(\Gamma)$. We can then associate to $\mathcal{S}(\Gamma)$ a polytope, which is the space of possible assignments of dihedral angles to the three-dimensional simplices of $\mathcal{S}(\Gamma)$ so that, for each cell, the angles are compatible with Theorem 4.1. The definition takes into account which vertices of $\Gamma^{*}$ are intended to be ideal vertices. It is first written for an Euclidean metric with conical singularities. 
Definition 5.1 Let $\Gamma$ be the one-skeleton of a cellular decomposition of a closed surface $\Sigma$, and let $V \subset \Gamma_{2}$ be a subset of the set of faces of $\Gamma$. We call $\mathcal{A}_{E}(\Sigma, \Gamma)$ the space of assignments, to each of the cells of $\mathcal{S}(\Gamma)$ associated to the vertices of $\Gamma$, of a set of dihedral angles, satisfying the conditions given in Theorem 4.1, so that, for each cell:

- the sum of the dihedral angles of the vertical edges is equal to $2 \pi$, and

- for each $v$ vertex other than the central vertex, the sum of the angles assigned to the adjacent edges is equal to $2 \pi$ if $v \in V$, and strictly larger than $2 \pi$ otherwise.

A natural complement is the definition of a space of assignments of angles to the edges of $\mathcal{S}(\Gamma)$ - of course this space has much lower dimension than $\mathcal{A}_{E}(\Sigma, \Gamma)$, since each edge of $\mathcal{S}(\Gamma)$ can be an edge of several simplices of that same simplicial complex.

Definition 5.2 Let $\Gamma$ be the one-skeleton of a cellular decomposition of $\Sigma$.

- We call $\mathcal{D}^{\prime}(\Gamma)$ the space of functions from the set of edges of $\mathcal{S}(\Gamma)$ to $\mathbb{R}$.

- We call $\sigma$ the linear map from $\mathcal{A}_{E}(\Sigma, \Gamma)$ to $\mathcal{D}^{\prime}(\Gamma)$, such that, for each $\Theta \in$ $\mathcal{A}_{E}(\Sigma, \Gamma)$ and each edge $e$ of $\mathcal{S}(\Gamma)$, the value of $\sigma(\Theta)$ on $e$ is equal to the sum of the angles at $e$ of the cells of $\mathcal{S}(\Gamma)$ containing it.

Note that $\mathcal{A}_{E}(\Sigma, \Gamma)$ is defined by linear equalities and inequalities, so that it is the intersections of a finite number of half-spaces in $\mathbb{R}^{p}$, for some $p$. Moreover $\mathcal{A}_{E}(\Sigma, \Gamma)$ is relatively compact, since the angles are in $(0, \pi)$, so it's closure is a polytope. It follows that its image by $\sigma$ is also a polytope in $\mathcal{D}^{\prime}(\Gamma)$. Definition 5.1 has a variant for surfaces with singular hyperbolic metrics:

Definition 5.3 Let $\Sigma$ be a closed surface, let $\Gamma$ be the one-skeleton of a cellular decomposition of $\Sigma$, and let $V \subset \Gamma_{2}$ be a subset of the set of faces of $\Gamma$. We call $\mathcal{A}_{H}(\Sigma, \Gamma)$ the space of assignments, to each of the cells of $\mathcal{S}(\Gamma)$ associated to the vertices of $\Gamma$, of a set of dihedral angles, satisfying the conditions given in Theorem 4.1, so that, for each cell:

- the sum of the dihedral angles of the vertical edges is strictly larger than $2 \pi$, and

- for each vertex $v$ other than the central vertex, the sum of the angles assigned to the adjacent edges is equal to $2 \pi$ if $v \in V$, and strictly larger otherwise.

\subsection{Gluing Conditions}

An angle assignment on the simplices of $\mathcal{S}(\Gamma)$, for a graph embedded in a surface, does not always define a hyperbolic metric on $\mathcal{S}(\Gamma)$. There are basically two reasons for this; the first is that it might not be possible to glue isometrically the faces of the simplices, the second is that, if such a gluing is possible, some nasty singularities might appear along the edges of $\mathcal{S}(\Gamma)$.

The Possibility of Gluing The next definition is quite natural, it should be compared to the definition of term "consistent" above. 
Definition 5.4 Let $\Gamma$ be the one-skeleton of a cellular decomposition of a closed surface $\Sigma$, and let $\Theta \in \mathcal{A}_{E}(\Sigma, \Gamma) . \Theta$ is compatible if the hyperbolic metrics on the simplices of $\mathcal{S}(\Gamma)$ can be glued along the vertical faces to yield a (non-complete) hyperbolic metric on the complement of the edges of $\mathcal{S}(\Gamma)$.

The same definition will be used in the context of circle patterns on closed hyperbolic surfaces, as well as for Euclidean and hyperbolic circle patterns on the disk; we do not repeat the definition.

More on the Possible Singularities A compatible angle assignment $\Theta$ does not, in general, define a hyperbolic metric which can be extended to the edges of $\mathcal{S}(\Gamma)$, since the holonomy around those edges could be nontrivial. More precisely, each edge $e$ of $\mathcal{S}(\Gamma)$ corresponds to a segment of geodesic for the hyperbolic metrics on each of the simplices which contain it, and the holonomy of a curve going around this edge can be decomposed as the sum of a translation along $e$ and a rotation around $e$. The translation component of the holonomy around $e$ can be obtained as follows. Let $S_{1}, \ldots, S_{p}$ be the three-cells containing $e$, in the cyclic orders in which they stand around $e$ (with $S_{p+1}:=S_{1}$ ). Let $j \in\{1, \ldots, p\}$, so that $S_{j}$ and $S_{j+1}$ share a face $f_{j}$. Let $H_{1}$ be a totally geodesic plane in $S_{1}$ which is orthogonal to $e$. Then $H_{1}$ can be extended uniquely across $f_{1}$ to a totally geodesic plane $H_{2}$ in $S_{2}$, orthogonal to $e$. Repeating this procedure, we obtain totally geodesic planes $H_{3}, \ldots, H_{p}$, all orthogonal to $e$. The translation component along $e$ of the holonomy around $e$ is then the oriented distance, along $e$, between $e \cap H_{1}$ and $e \cap H_{p}$. The same holds with the totally geodesic planes replaced by horospheres.

In addition, the holonomy on elements of the fundamental group of the surface can also be "complicated", as should be apparent from the definition of a "consistent" metric in Definition 4.10.

The case of circle patterns on hyperbolic surfaces is much simpler, because, as will be apparent in the following, the translation component of the holonomy is always zero, so that the noncomplete metric obtained by gluing the hyperideal simplices (when it is possible) can always be completed to a hyperbolic metric with cone singularities along lines (and with polyhedral boundary).

\subsection{Hyperbolic Circle Patterns on Closed Surfaces}

The description just made in the hyperbolic case can be translated as the following lemma.

Lemma 5.5 Let $\Sigma$ be a closed surface, and let $\Gamma$ be the one-skeleton of a cellular decomposition of $\Sigma$, with trivalent vertices. Let $\Theta \in \mathcal{A}_{H}(\Sigma, \Gamma)$. If $\Theta$ is compatible, then it defines a hyperbolic metric on $\mathcal{S}(\Gamma)$ with conical singularities along the edges.

Proof Let $S_{1}, \ldots, S_{N}$ be the simplices in $\mathcal{S}(\Gamma)$. For each $i=1, \ldots, N$, there exists a unique hyperbolic plane $H_{i}$ in $S_{i}$ which is dual to the "central" vertex of $\mathcal{S}(\Gamma)$. It is characterized by the fact that it intersects orthogonally all the edges adjacent to the "central" vertex of $S_{i}$. 
Suppose that $S_{i}$ and $S_{j}$ are two simplices in $\mathcal{S}(\Gamma)$ which share a two-dimensional face, $f$. The intersections of $H_{i}$ and of $H_{j}$ with $f$ are two geodesic segments which both intersect orthogonally the two edges of $f$ adjacent to the "central" vertex. It follows that $H_{i} \cap f=H_{j} \cap f$.

Let $e$ be a vertical edge of $\mathcal{S}(\Gamma)$. Then the description given above of the translation component of the holonomy around $e$ can be applied with each $H_{i}$ equal to the totally geodesic plane, in $S_{i}$, dual to the "central" vertex $v_{0}$; indeed those planes are orthogonal to $e$, and $H_{i} \cap e=H_{i+1} \cap e$, as needed. It follows immediately that the translation component of the holonomy around $e$ vanishes, so that the hyperbolic metric defined by $\Theta$ can be completed to a hyperbolic metric with conical singularities along the vertical edges of $\mathcal{S}(\Gamma)$.

The conditions under which the simplices of $\mathcal{S}(\Gamma)$ can be glued-according to Proposition 4.2, that the length of the edges of each "vertical" face is the same for both simplices containing it—can be expressed quite simply in the hyperbolic case. Indeed, each three-cell of $\mathcal{S}(\Gamma)$ contains a hyperbolic plane which is dual to $v_{0}$, so there is a canonical choice of the horospheres centered at the ideal vertices-they can be chosen as the one which are tangent to this dual plane.

There is a natural function defined over $\mathcal{A}_{H}(\Sigma, \Gamma)$ : the sum of the hyperbolic volumes of the simplices in $\mathcal{S}(\Gamma)$, for the hyperbolic metrics determined by the dihedral angles which are assigned to each of them. Note that those hyperbolic metrics are the metrics on hyperideal simplices, so that the volume which is used has to be the volume of the corresponding truncated simplices. We call $\mathcal{V}$ this total volume.

Lemma 5.6 Let $\Theta \in \mathcal{A}_{H}(\Sigma, \Gamma)$ be an assignment of angles to the simplices in $\mathcal{S}(\Gamma)$, and let $\alpha:=\sigma(\Theta)$. Then $\Theta$ is compatible if and only if $\Theta$ is a critical point of the restriction of $\mathcal{V}$ to $\sigma^{-1}(\alpha) \subset \mathcal{A}_{H}(\Sigma, \Gamma)$.

Proof Suppose first that $\Theta$ is a compatible, and choose for each ideal vertex of each three-cell of $\mathcal{S}(\Gamma)$ a horosphere as suggested above, that is, tangent to the hyperbolic plane which is dual to the "central" vertex $v_{0}$.

Consider a "vertical" edge $e$ of $\mathcal{S}(\Gamma)$, such that the endpoint of $e$ other than $v_{0}$ is an ideal vertex $v$ of $\mathcal{S}(\Gamma)$. Then the hyperbolic planes dual to $v_{0}$ in each of the threecells containing $e$ intersect $e$ at the same point, say $p$, where they are orthogonal to $e$. The horospheres centered at $v$ in each of those three-cells are also orthogonal to $e$; since they are tangent to planes dual to $v_{0}$, they have to intersect $e$ at $p$. It follows that the length of $e$ is 0 in each of the three-cells that contain it (relative to the choice of the horospheres tangent to the planes dual to $v_{0}$ ). According to Proposition 4.2, the length of each "horizontal" edges of $\mathcal{S}(\Gamma)$, for the hyperbolic metrics on each of the two three-cells containing it (and for this choice of horospheres) have to be equal.

Let $\Theta^{\prime}$ be a first-order deformation of $\Theta$ in $\sigma^{-1}(\alpha)$. In other terms, to each threecell $S$ of $\mathcal{S}(\Gamma)$ and each edge $e$ of $S$ is associated a number, $\Theta^{\prime}(S, e)$, corresponding to the first-order variation of the dihedral angle of $S$ at $e$, and the $\Theta^{\prime}(S, e)$ satisfy some linear constraints corresponding to the ideal vertices (the vertices in $V$ ). The fact that $\Theta^{\prime}$ is tangent to $\sigma^{-1}(\alpha)$ then means that the sum of the numbers attached to an edge, for each of the three-cells containing it, vanishes. 
It follows from the Schläfli formula (5) that

$$
d \mathcal{V}\left(\Theta^{\prime}\right)=\sum_{S \subset \mathcal{S}(\Gamma)} \sum_{e \subset S} l_{S}(e) \Theta^{\prime}(S, e),
$$

where the first sum is over the three-cells in $\mathcal{S}(\Gamma)$ and the second over the edges in each three-cells, and $l_{S}(e)$ is the length of the edge $e$ for the metric in the cell $S$. But we have seen that $l_{S}(e)$ does not depend on $S$ and can be written as $l(e)$, so that the sum above can be written as

$$
d \mathcal{V}\left(\Theta^{\prime}\right)=\sum_{e} l(e) \sum_{S \supset e} \Theta^{\prime}(S, e)
$$

which makes it apparent that $d \mathcal{V}\left(\Theta^{\prime}\right)=0$.

Conversely, suppose that $\Theta$ is not compatible, which means that it is not possible to glue isometrically the simplices in $\mathcal{S}(\Gamma)$ along their common faces. If there is an edge $e$ of $\mathcal{S}(\Gamma)$ between two strictly hyperideal vertices, which has different lengths in two simplices $S$ and $S^{\prime}$ which contain it, then we can consider the first-order variation of the angles which increases at unit speed the angle of $S$ at $e$ and decreases at unit speed the angle of $S^{\prime}$ at $e$, while keeping all other angles fixed. The Schläfli formula shows that, under this deformation, the first-order variation of $\mathcal{V}$ is nonzero. So we can now suppose that the edges between strictly hyperideal vertices are the same in all the simplices containing them.

Choose horospheres centered at the ideal vertices of $\mathcal{S}(\Gamma)$ as above, i.e. tangent to the hyperbolic planes dual to $v_{0}$. Then the same argument as above clearly shows that the lengths of the "vertical" edges of $\mathcal{S}(\Gamma)$ are zero in each of the cells that contain them so, by Proposition 4.2, there is at least one "horizontal" edges of $\mathcal{S}(\Gamma)$, $e_{0}$, which has different lengths in the two cells containing it, say $S_{1}$ and $S_{2}$.

Let $v_{1}$ and $v_{2}$ be the endpoints of $e_{0}$. Consider the first-order variation $\Theta^{\prime}$ of $\Theta$ which is defined as follows:

- the angle of $S_{1}$ at $e$ has derivative 1 , and the angle of $S_{2}$ at $e$ has derivative -1 ;

- the angle of $S_{1}$ on the "vertical" edge of $\mathcal{S}(\Gamma)$ ending at $v_{1}$ has derivative -1 , as the angle of $S_{1}$ on the "vertical" edge of $\mathcal{S}(\Gamma)$ ending at $v_{2}$;

- the angle of $S_{2}$ on the "vertical" edge of $\mathcal{S}(\Gamma)$ ending at $v_{1}$ has derivative 1 , as the angle of $S_{2}$ on the "vertical" edge of $\mathcal{S}(\Gamma)$ ending at $v_{2}$; and

- the other angles remain constant.

Then $\Theta^{\prime}$ is a tangent vector to $\mathcal{A}_{H}(\Sigma, \Gamma)$, because the only linear constraints on the first-order variations of the angles of the simplices is that the sum of the angles in each cell of the edges adjacent to an ideal vertex remain constant, and it happens here for both $v_{1}$ and $v_{2}$ (which might or might not be ideal).

Moreover, $\Theta^{\prime}$ is tangent to $\sigma^{-1}(\alpha)$, because it is easy to check that the sum of the angles remain fixed at $e_{0}$ and at the vertical edges ending at $v_{1}$ and at $v_{2}$. However the Schläfli formula shows that $d \mathcal{V}\left(\Theta^{\prime}\right) \neq 0$, with the only nonzero term in (6) coming from $e_{0}$. This finishes the proof.

The key infinitesimal rigidity statement that we need follows from the previous lemma and from the strict concavity of $\mathcal{V}$, considered as a function of the dihedral 
angles of the simplices in $\mathcal{S}(\Sigma, \Gamma)$. We say here that a dual circle is "ideal" if it is reduced to a point.

Lemma 5.7 Let $\Sigma$ be a closed surface, and let $C$ be a hyperideal circle pattern on $\Sigma$, with respect to a hyperbolic metric with conical singularities at the center of the dual circles. The first-order deformations of $C$, among hyperideal circle patterns with the same incidence graph and the same set of ideal dual circles, are parameterized by the first-order variations of the intersection angles between the circles and of the singular curvatures at the centers of the dual circle $c^{\prime}$, under the condition that, for each ideal dual circle, the sum of the intersection angles between adjacent principal circles varies as the singular curvature at $c^{\prime}$.

Proof The constructions made in Sect. 4, and in particular Proposition 4.13, show that the statement of the lemma is equivalent to another statement on the angle assignments to the simplices of $\mathcal{S}(\Sigma, \Gamma)$, where $\Gamma$ is the incidence graph of $C$. Namely that, given $\Theta \in \mathcal{A}_{H}(\Sigma, \Theta)$ which is compatible, the first-order deformations of $\Theta$ among compatible angle assignments are uniquely determined by the corresponding first-order variations of their images in $\mathcal{D}^{\prime}(\Sigma, \Gamma)$ by $d \sigma$. The fact that each ideal dual circle $c^{\prime}$ remains ideal is a consequence of the fact that the sum of the intersection angles between the adjacent principal circles varies as the singular curvature at $c^{\prime}$.

Since $\sigma$ is a linear map, the inverse images by $\sigma$ of the elements of $\mathcal{D}^{\prime}(\Sigma, \Gamma)$ define a foliation of $\mathcal{A}_{H}(\Sigma, \Gamma)$ by affine submanifolds. Since $\Theta$ is compatible, it is a critical point of $\mathcal{V}$ restricted to the inverse image by $\sigma$ of $\sigma(\Theta)$ by Lemma 5.6. Since $\mathcal{V}$ is strictly concave over $\mathcal{A}_{H}(\Sigma, \Gamma)$ - as a sum of the volumes of the simplices, which are strictly concave functions by Lemma $4.4-\Theta$ is a local maximum of $\mathcal{V}$, and each leaf close to $\Theta$, i.e. the inverse image by $\sigma$ of each element $\beta$ of $\mathcal{D}^{\prime}(\Sigma, \Gamma)$ close to $\sigma(\Theta)$, contains a unique maximum of the restriction of $\mathcal{V}$. Moreover, the inverse function theorem, along with the strict concavity of $\mathcal{V}$, shows that this local maximum varies smoothly with $\beta$. The result follows.

\subsection{Euclidean Circle Patterns and the Holonomy}

We now turn to hyperideal circle patterns on a surface with a singular Euclidean metric. As mentioned earlier, the argument is basically similar to what has just been said on the case of hyperbolic surfaces, but with some additional arguments which are necessary to understand the translation component of the holonomy around "vertical" edges of $\mathcal{S}(\Gamma)$, as well as when the hyperbolic metric is consistent (cf. Definition 4.10). It is in particular helpful to consider a special kind of first-order variation of the dihedral angles of the simplices, as explained in the next paragraph.

Some Convenient Deformations Let $\gamma$ be an oriented simple closed path in $\Gamma$. Consider an angle assignment $\Theta \in \mathcal{A}_{E}(\Sigma, \Gamma)$. We associate to $\gamma$ a first-order deformation $\Theta_{\gamma}^{\prime}$ of $\Theta$ (i.e. a vector tangent to $\mathcal{A}_{E}(\Sigma, \Gamma)$ at $\Theta$ ) as explained in Fig. 9; the closed curve $\gamma$ appears as the thicker polygonal curve. Each triangle corresponds to a simplex in $\mathcal{S}(\Sigma, \Gamma)$, each segment to a "horizontal" edge, and each vertex to a "vertical" edge of $\mathcal{S}(\Sigma, \Gamma)$; the signs "+" are attached to edges with an angle, in the simplex on which the sign stands, which has differential +1 , while the signs "-" are 
Fig. 9 A useful deformation associated with a closed path in $\Gamma$

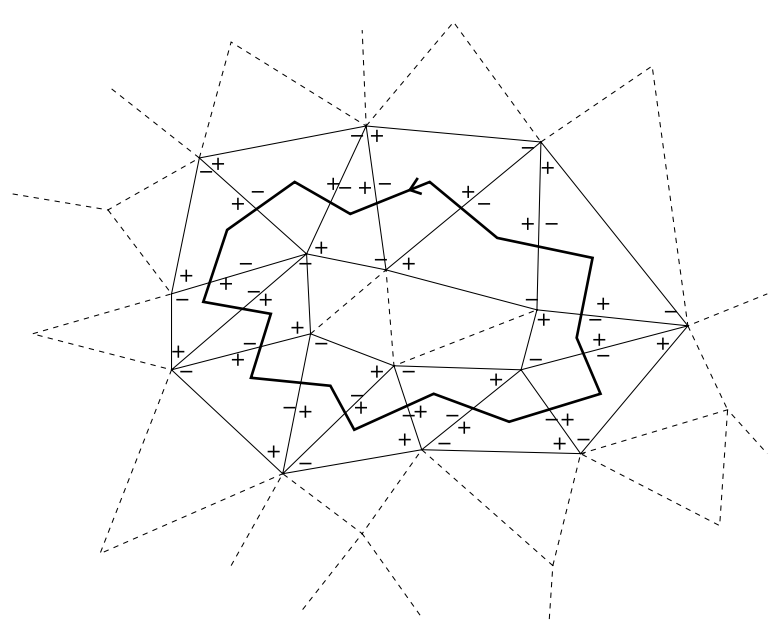

attached to edges with an angle with differential -1 . The edges which have no sign attached have an angle which remains constant.

A more explicit description of $\Theta_{\gamma}^{\prime}$ can be given as follows. Let $v_{1}, \ldots, v_{N}$ be the successive vertices of $\gamma$, which correspond to faces $v_{1}^{*}, \ldots, v_{N}^{*}$ of $\Gamma^{*}$ and therefore to simplices $S_{1}, \ldots, S_{N}$ of $\mathcal{S}(\Gamma)$. The union of the interiors of the triangles $v_{i}^{*}, i=$ $1, \ldots, N$, along with the edges $e_{i}$ of $\Gamma^{*}$ between $v_{i}^{*}$ and $v_{i+1}^{*}, i=1, \ldots, N$, is an open strip, which is called $S \cup$ here. Its boundary $\partial S_{\cup}$ has two connected components, $\partial_{l} S_{\cup}$ and $\partial_{r} S_{\cup}$, respectively, on the left and on the right of $\gamma$. We parameterized them with the orientation coming from the orientation of $\gamma$, that is, the orientation of $\partial_{r} S_{\cup}$ is compatible with the orientation of $S_{\cup}$, but the orientation of $\partial_{l} S_{\cup}$ is opposite to the natural orientation of $\partial S_{\cup}$. Note that both $\partial_{l} S \cup$ and $\partial_{r} S_{\cup}$ can, in some cases, be reduced to points.

Let $i \in\{1, \ldots, N\}$. The edge $e_{i}$ between $v_{i}^{*}$ and $v_{i+1}^{*}$ corresponds to a "horizontal" edge $e_{i}^{\prime}$ of $\mathcal{S}(\Gamma)$ which is contained in two simplices of $\mathcal{S}(\Gamma), S_{i}$ and $S_{i+1}$. Under the deformation $\Theta_{\gamma}^{\prime}$, the angle of $S_{i}$ at $e_{i}^{\prime}$ has differential equal to 1 , and the angle of $S_{i+1}$ at $e_{i}^{\prime}$ has differential equal to -1 . Moreover, the triangle $v_{i}^{*}$ has two edges which are contained in the interior of $S_{\cup}$, namely $e_{i-1}$ and $e_{i}$, and exactly one edge, say $E_{i}$, which is either on $\partial_{l} S_{\cup}$ or on $\partial_{r} S_{\cup}$. In each case, we call $E_{i,-}$ and $E_{i,+}$ the endpoints of $E_{i}$, ordered according to the orientation of $\partial_{l} S_{\cup}$ and of $\partial_{r} S \cup$ described above. Then $E_{i,-}$ (resp., $\left.E_{i,+}\right)$ corresponds to a "vertical" edge $E_{i,-}^{\prime}$ (resp., $E_{i,+}^{\prime}$ ), which is contained in several simplices of $\mathcal{S}(\Gamma)$, including $S_{i}$. The angle of $S_{i}$ at $E_{i,+}^{\prime}$ has differential equal to 1 , and the angle of $S_{i}$ at $E_{i,-}^{\prime}$ has differential equal to -1 . The same holds for all $i \in\{1, \ldots, N\}$, and the other angles remain constant.

It is necessary to check that the first-order variation of the angles of the simplices of $\mathcal{S}(\Gamma)$ indeed defines a vector tangent to $\mathcal{A}_{E}(\Sigma, \Gamma)$ at $\Theta$, i.e. that the linear constraints on the angles of the simplices remain satisfied. Since those constraints correspond to the ideal vertices of the simplices of $\mathcal{S}(\Gamma)$, the result follows by considering three cases.

- At the "central" vertex $v_{0}$ (which is ideal since we're considering Euclidean metrics). Each $S_{i}, i=1, \ldots, N$, has exactly two edges ending at $v_{0}$ on which the angles 
vary, namely $E_{i,-}^{\prime}$ and $E_{i,+}^{\prime}$. Since the differentials of those angles are opposite, the sum of the angles of the edges of $S_{i}$ adjacent to $v_{0}$ remains equal to $2 \pi$.

- Let $v$ be a vertex of $\partial_{l} S_{\cup}$ (the same argument can be used for a vertex of $\left.\partial_{r} S_{\cup}\right)$. Then $v$ is the endpoint of an even number $2 p$ of edges of $\partial_{l} S_{\cup}$, say $E_{i_{1}}, E_{i_{2}}, \ldots, E_{i_{2 p}}$. So

$$
v=E_{i_{1},+}=E_{i_{2},-}=E_{i_{3},+}=E_{i_{4},-}=\cdots=E_{i_{2 p-1},+}=E_{i_{2 p},-} .
$$

There are two kinds of simplices of $\mathcal{S}(\Gamma)$ containing edges on which the angle varies under the deformation $\Theta^{\prime}(\gamma)$ :

- the simplices $S_{i_{2 k-1}}$ (resp., $S_{i_{2 k}}$ ), $k=1, \ldots, p$, on which the angle on the "vertical" edge ending at $v$ has differential equal to 1 (resp., -1 ), while the angle on the "horizontal" edge $e_{i_{2 k-1}+1}^{\prime}$ (resp., $e_{i_{2 k}}^{\prime}$ ) has differential -1 (resp., 1); and

- the other simplices corresponding to triangles $v_{i}^{*}$ which contain $v$ but have no edge containing $v$ and contained in $\partial_{l} S_{\cup}$. The angles of those simplices at edges containing $v$ are constant except for two horizontal edges, on which the differentials of the angles are opposite.

- At all vertices of $\mathcal{S}(\Gamma)$ which are neither $v_{0}$ nor a vertex of $\partial S_{\cup}$, no angle varies.

This shows that $\Theta^{\prime}(\gamma)$ defines a vector tangent to $\mathcal{A}_{E}(\Sigma, \Gamma)$.

In addition, the first-order variation that we consider does not vary the total angle around the "vertical" edges of $\mathcal{S}(\Gamma)$ and the dihedral angles at the "horizontal" edges.

Proposition 5.8 Let $\alpha:=\sigma(\Theta)$. Then $\Theta^{\prime}(\gamma)$ is tangent to $\sigma^{-1}(\alpha)$.

Proof The proof should be apparent from Fig. 9. On the "horizontal" edges of $\mathcal{S}(\Gamma)$ which are not one of the $e_{i}^{\prime}$, none of the angle varies, so the total angle remains constant. On the $e_{i}^{\prime}, i=1, \ldots, N$, the two angles vary in opposite ways, so that again the total angle remains constant.

Similarly, for "vertical" edges of $\mathcal{S}(\Gamma)$, no angle varies except when one of the endpoints is a vertex of $\partial S_{\cup}$. When one of the endpoints is on $\partial S_{\cup}$ (the other being $v_{0}$, as for all "vertical" edges) there is a even number of angles which vary, half of them with differential 1 and the other with differential -1 , and the sum of the angles remains constant, as claimed.

A simple but already useful instance of a deformation is obtained by taking as $\gamma$ the boundary of a face of $\Gamma$. Then $\partial_{l} S_{\cup}$ is reduced to a point.

Critical Points of the Volume Functional We are now equipped to prove the Euclidean version of the key technical point of this subsection: the critical points of the restriction of $\mathcal{V}$ to the level sets of $\sigma$ are the angle assignments for which the simplices of $\mathcal{S}(\Gamma)$ can be isometrically glued along their faces, in such a way that it possible to choose horosphere centered at all "ideal" vertices which "match" along the two-dimensional faces.

Lemma 5.9 Let $\Theta \in \mathcal{A}_{E}(\Sigma, \Gamma)$, and let $\alpha:=\sigma(\Theta)$. Then $\Theta$ is a critical point of the restriction of $\mathcal{V}$ to $\sigma^{-1}(\alpha)$ if and only if: 
Fig. 10 Deformations close to a face without gluing, with two (left) or one (right) ideal vertices
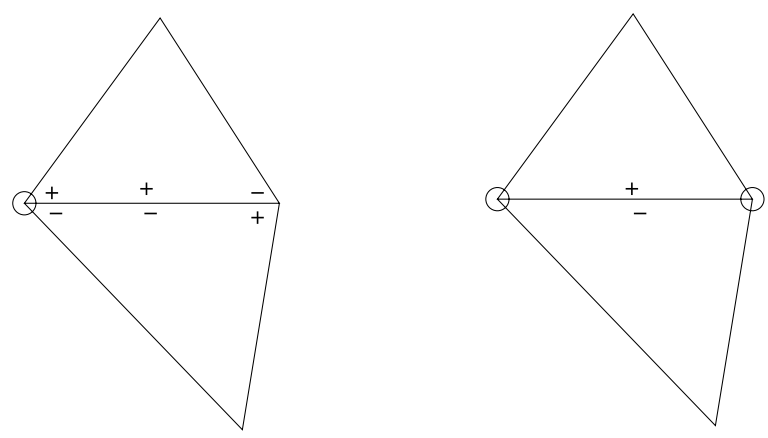

1. the hyperbolic metrics defined by $\Theta$ on the simplices of $\mathcal{S}(\Gamma)$ can be glued isometrically along their faces, and

2. the resulting hyperbolic metric can be completed on the "vertical" edges of $\mathcal{S}(\Gamma)$ to obtain a hyperbolic with conical singularities on $\mathcal{S}(\Gamma)$ which moreover is consistent.

Proof Suppose first that conditions (1) and (2) hold. Let $h$ be the hyperbolic metric with conical singularities obtained by gluing the hyperbolic metrics on the simplices of $\mathcal{S}(\Gamma)$. Then, by definition of a consistent hyperbolic metric (with conical singularities) it is possible to choose for each simplex $S$ of $\mathcal{S}(\Gamma)$ a horosphere $H(S)$ centered at the "central" vertex $v_{0}$ in such a way that, if $S$ and $S^{\prime}$ are two simplices which share a face $f$, then $H(S) \cap f=H\left(S^{\prime}\right) \cap f$. It is then also possible to choose, for each ideal vertex $v$ of $\mathcal{S}(\Gamma)$ (other than $v_{0}$ ) and each simplex $S$ adjacent to $v$ a horosphere $H^{\prime}(S, v)$ centered at $v$, which is tangent to $H(S)$, and this condition defines $H^{\prime}(S, v)$ uniquely. Moreover, if $S$ and $S^{\prime}$ are two simplices which share a face $f$ containing $v$, then $H^{\prime}(S, v) \cap f=H^{\prime}\left(S^{\prime}, v\right) \cap f$, because both curves are horocycles which are tangent at the edge $\left[v_{0}, v\right]$ to $H(S) \cap f=H\left(S^{\prime}\right) \cap f$.

Let $\Theta^{\prime}$ be a first-order variation of $\Theta$ which is tangent to $\sigma^{-1}(\alpha)$. The same argument as in the proof of Lemma 5.6, with this choice of horospheres, shows that $d \mathcal{V}\left(\Theta^{\prime}\right)=0$. So conditions (1) and (2) imply that $\Theta$ is a critical point of the restriction of $\mathcal{V}$ to $\sigma^{-1}(\alpha)$.

Conversely, suppose first that condition (1) does not hold. This means that there is a "vertical" face $f$ of $\mathcal{S}(\Gamma)$, between two simplices which cannot be isometrically glued. Since two ideal triangles are always isometric, $f$ has either one or two ideal vertices (including $v_{0}$ ). Figure 10 describes a simple first-order variation of the angles of the simplices containing $f$ which leads to a nonzero variation of the sum of the volumes of the two simplices; the strictly hyperideal vertices are marked with circles.

Checking that it is indeed the case is quite obvious when $f$ has no ideal vertex beyond $v_{0}$ (on the right in Fig. 10), since there is no linear constraint at the strictly hyperideal vertices, and the fact that the total volume varies is a direct consequence of the Schläfli formula, since the length for the two metrics of the "horizontal" edge in $f$ has to be different.

Now suppose that $f$ has two ideal vertices at $v_{0}$ and $v_{1}$, and a strictly hyperideal vertex at $v_{2}$ (pictured on the left in Fig. 10). Note that the deformation pictured there is indeed tangent to $\mathcal{A}_{E}(\Sigma, \Gamma)$, because the linear constraints at $v_{0}$ and at $v_{1}$ hold. It 
is helpful to choose, in both simplices, horospheres centered at $v_{0}$ which are tangent to the hyperbolic plane dual to $v_{2}$, and horospheres centered at $v_{1}$ which are tangent to the horospheres centered at $v_{0}$. This way the length of the "vertical" edges of $f$ are zero, and it is clear that the deformations pictured in Fig. 10 induces a nonzero firstorder variation of the sum of the volumes of the simplices. Finally, this deformation is tangent to $\sigma^{-1}(\alpha)$, because the sum of the angles at all three edges of $f$ remains constant.

The next step is when $\Theta$ defines hyperbolic metrics on the simplices of $\mathcal{S}(\Gamma)$ which can be isometrically glued, but so that the translation component of the holonomy around one of the "vertical" edges of $\mathcal{S}(\Gamma)$, say $e$, is nonzero. In other terms, it is possible to choose horosphere centered at $v_{0}$ for each of the simplices $S_{1}, \ldots, S_{N}$ containing $e$, so that the horospheres on both sides of $S_{i} \cap S_{i+1}$ match for $i=1, \ldots, N-1$, but not for $i=N$. The endpoints of $e$ are $v_{0}$ and a vertex $v$ of $\Gamma^{*}$, corresponding to a face $v^{*}$ of $\Gamma$. Consider the first-order variation $\Theta_{\partial v^{*}}^{\prime}$. Proposition 5.8 shows that it is tangent to $\sigma^{-1}(\alpha)$, while the Schläfli formula shows that $d \mathcal{V}\left(\Theta_{\partial v^{*}}^{\prime}\right) \neq 0$.

The last step is to suppose that the hyperbolic metrics on the simplices can be glued, that the resulting metric on $\mathcal{S}(\Gamma)$ minus its edges has zero translation holonomy around the "vertical" edges, but that it is not consistent. Definition 4.10 then means that it is not possible to choose horospheres centered at $v_{0}$, in all the simplices of $\mathcal{S}(\Gamma)$, in such a way that their intersections with the "vertical" faces match. This means that there exists a closed path $\gamma$ in $\Gamma$ (which has to be homotopically nontrivial), with vertices $v_{1}, \ldots, v_{N}$ corresponding to simplices $S_{1}, \ldots, S_{N}$ of $\mathcal{S}(\Gamma)$, so that, if one choose horospheres $H_{1}, \ldots, H_{N}$ centered at $v_{0}$ in the $S_{i}$ such that their intersections with $S_{1} \cap S_{2}, S_{2} \cap S_{3}, \ldots, S_{N-1} \cap S_{N}$ match, then the intersections of the horosphere $H_{N}$ in $S_{N}$ with $S_{N} \cap S_{1}$ does not match with the intersection with that same face of the horosphere $H_{1}$ in $S_{1}$; let $\delta$ be the oriented distance between them.

For each ideal vertex $v$ of the $S_{i}$ other than $v_{0}$, we choose horospheres such that for all $i=1, \ldots, N$, if $v \in S_{i} \cap S_{i+1}$ then the horospheres chosen at $v$ in $S_{i}$ and in $S_{i+1}$ match on $S_{i} \cap S_{i+1}$.

We now use the Schläfli formula to compute the variation $d \mathcal{V}\left(\Theta_{\gamma}^{\prime}\right)$ under the firstorder deformation $\Theta_{\gamma}^{\prime}$ of the angles assigned to the simplices of $\mathcal{S}$. With the choice of horospheres described it is quite clear that the contributions corresponding to all the edges except the two "vertical" edges of $S_{1} \cap S_{N}$ cancel out, so that $d \mathcal{V}\left(\Theta^{\prime}(\gamma)\right)=$ $-2 \delta \neq 0$.

Euclidean Circle Patterns The results of the previous paragraph lead naturally to an analog, for Euclidean surfaces with conical singularities, of Lemma 5.7.

Lemma 5.10 Let $C$ be a hyperideal circle pattern on a closed surface $\Sigma$, with respect to a Euclidean metric with conical singularities at the center of the dual circles. The first-order deformations of $C$, among hyperideal circle patterns with the same incidence graph and the same set of ideal dual circles (considered up to homothety) are parameterized by the first-order variations of the intersection angles between the circles and of the singular curvatures at the centers of the dual circles, under the condition that, for each ideal dual circle $c^{\prime}$, the sum of the intersection angles between the adjacent principal circles varies as the singular curvature at $c^{\prime}$. 
The proof follows exactly the same pattern as the proof of Lemma 5.7, the basic block is now Lemma 5.9 .

\section{Compactness}

This section deals with the properness of the map sending a hyperideal circle pattern on a singular surface to its intersection angles and singular curvatures. This can be stated in terms of compactness: a sequence of such circle patterns, with given incidence graph and such that the intersection angles and the singular curvatures converge to a "reasonable" limit, has a subsequence which converges to a "good" circle pattern.

The methods used here are elementary, based solely on considering the possible behavior of the circles relative to the underlying metric. Other approaches are possible, in particular using the three-dimensional geometry of the hyperbolic simplicial complex associated to a circle pattern.

\subsection{Statement}

We state here the compactness lemma which is used in the proofs of the main theorems.

Lemma 6.1 Let $\Gamma$ be a graph embedded in the closed surface $\Sigma$, and let $\left(C_{n}\right)_{n \in \mathbb{N}}$ be a sequence of hyperideal circle patterns with incidence graph $\Gamma$, on $\Sigma$ with a sequence of metrics $\left(h_{n}\right)_{n \in \mathbb{N}}$ of constant curvature $K_{n} \leq 0$, of area equal to 1 , with conical singularities at the centers of the dual circles. Suppose that $K_{n} \rightarrow K_{\infty}$, and that the intersection angles and the singular curvatures are described by functions $\theta_{n}: \Gamma_{1} \rightarrow(0, \pi)$ and $\kappa_{n}: \Gamma_{2} \rightarrow(-\infty, 2 \pi)$, which are converging towards functions $\theta: \Gamma_{1} \rightarrow(0, \pi)$ and $\kappa: \Gamma_{2} \rightarrow \mathbb{R}$, respectively, such that $\theta$ and $\kappa$ still satisfy the hypothesis of Theorem 1.4 or 1.6 (depending on whether $K_{\infty}=0$ or $K_{\infty}<0$ ). Then, perhaps after extracting a subsequence, $\left(C_{n}\right)_{n \in \mathbb{N}}$ converges to a circle pattern $C$ on $\Sigma$, on a metric of constant curvature $K_{\infty}$ with conical singularities at the centers of the dual circles, with intersection angles given by $\theta$ and singular curvatures given bук.

The proof proceeds in two steps. The first is to prove that the radii of the principal circles remain bounded, i.e. they cannot become very small or very large. This implies that the distances between the singular points cannot become too small. Direct geometric arguments then show that those metrics with conical singularities have a converging subsequence.

\subsection{Collapsing Circles}

The following arguments use a graph which is canonically associated to $\Gamma$ in a simple manner.

Definition 6.2 Let $\Gamma^{s}$ the graph, embedded in $\Sigma$, which has: 
- one vertex for each vertex of $\Gamma$, and one for each vertex of $\Gamma^{*}$ (i.e. for each face of $\Gamma$ ), and

- one edge between two vertices if they correspond to adjacent vertices of $\Gamma$, or if they correspond to a face of $\Gamma$ and a vertex of $\Gamma$ contained in that face.

There is a simple interpretation of $\Gamma^{s}$, its vertices correspond to the circles which are either principal or dual circles in the circle pattern, and the edges correspond to pairs of adjacent circles. Clearly $\Gamma^{s}$ has triangular faces, with each face having one vertex corresponding to a dual circle and two corresponding to principal circles.

The proof of the compactness lemmas above is based on a simple proposition.

\section{Proposition 6.3 Under the hypothesis of Lemma 6.1, the radii of the principal circles} remain bounded between two positive constants.

Proof We have supposed that the area is always equal to 1 . But $\Gamma$ is fixed, so that the valence of its vertices is bounded, so that the number of self-intersections of a circle is also bounded from above. It follows quite directly that the radii of the circles cannot go to infinity. Suppose that, after taking a subsequence, the radii of some of the principal circles go to 0 . Then this also happens to one of the dual circle adjacent to each "collapsing" principal circle; indeed each principal circle is adjacent to at least three dual circles (because the principal circles correspond to the vertices of the embedded graph $\Gamma$ ). A "very small" principal circle has to be orthogonal to at least 3 dual circles, which are disjoint, and this is possible only if at least one of those adjacent dual circles is also very small.

Consider, in the graph $\Gamma^{s}$, the set $V_{0}$ of vertices corresponding to circles which have a radius going to 0 . The above remark shows that $V_{0}$ contains at least one point corresponding to a principal circle, and one corresponding to an adjacent dual circle. Let $\Gamma_{0}^{s}$ be the subgraph of $\Gamma^{s}$ which has as its vertices the vertices in $V_{0}$ and as edges the edges of $\Gamma^{s}$ with both endpoints in $V_{0}$, and let $\Gamma_{1}^{s}$ be a connected component of $\Gamma_{0}^{s}$ containing at least one vertex corresponding to a principal circle.

Let $\partial \Gamma_{1}^{S}$ be the set of edges of $\Gamma^{s}$ which are, in a face of $\Gamma^{s}$ with exactly one vertex in $\Gamma_{1}^{s}$, the edge opposite to that vertex. $\partial \Gamma_{1}^{s}$ is by construction an union of closed curves, which we call $\partial_{1} \Gamma_{1}^{s}, \ldots, \partial_{N} \Gamma_{1}^{s}$. By construction all the circles corresponding to the vertices of $\Gamma_{1}^{s}$ "collapse" to a point in the limit surface, while this happens to none of the circles correspondings to the vertices of $\partial \Gamma_{1}^{s}$.

We associate to $\Gamma_{1}$ an admissible domain $\Omega$ in $(\Sigma, \Gamma)$, as follows. For each edge $e$ of $\partial \Gamma_{1}^{s}$ with endpoints corresponding to vertices of $\Gamma$, we consider the corresponding edge $e^{\prime}$ of $\Gamma$. For each vertex $v$ of $\partial \Gamma_{1}^{s}$ corresponding to a face $f$ of $\Gamma$, the two vertices adjacent to $v$ in $\partial \Gamma_{1}^{s}$ correspond to vertices $v_{1}$ and $v_{2}$ of $\Gamma$, both contained in $f$. Then we consider a segment $s$ in $f$ going from $v_{1}$ to $v_{2}$.

Let $\gamma$ be the union of those two types of segments, it is a finite union of closed curves $\gamma_{1}, \ldots, \gamma_{N}$, with $\gamma_{i}$ corresponding to $\partial_{i} \Gamma_{1}^{S}$. It is also simple to check that $\gamma$ bounds a domain $\Omega$ in $\Sigma$, and that $\Omega$ is an admissible domain in $(\Sigma, \Gamma)$. Below we set $\partial_{i} \Omega:=\gamma_{i}$.

All vertices of $\Gamma_{1}^{s}$ correspond to circles which "collapse" to a point. Since $\Gamma_{1}^{s}$ is connected, all those circles actually "collapse" to the same point, which we call $x_{0}$. Since the circles corresponding to vertices of $\partial \Gamma_{1}^{s}$ have radii converging to positive 
Fig. 11 A collapsing annulus, and some of the adjacent circles

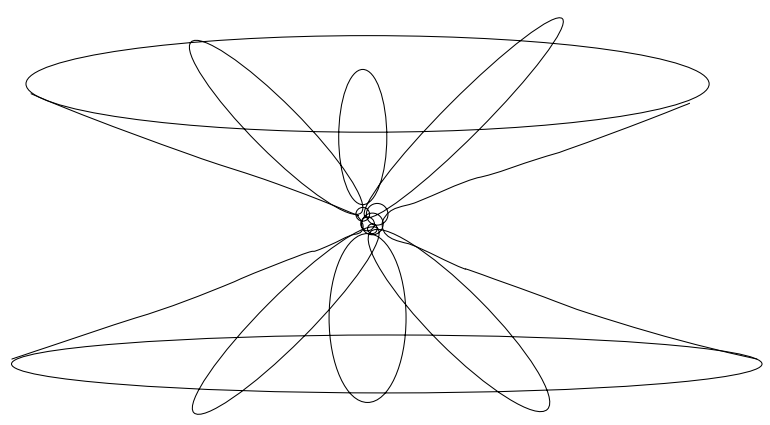

numbers, the union of the disks bounded by those circles converges to a flat surface $S_{1}$ with one singular point (which corresponds to $x_{0}$ ). More precisely, the limit surface is obtained by considering, for each connected component $\partial_{i} \Omega$ of $\partial \Omega$, a flat surface $S_{i}$ with boundary, with one conical singularity, at the point $x_{i}$ corresponding to $x_{0}$ under the gluing of those flat surfaces. Moreover, in each of the flat surfaces $S_{i}, 1 \leq i \leq N$, each of the circles contains the singular point.

For each $n \in \mathbb{N}$, let $\Omega_{n}$ be an open domain in $\left(\Sigma, h_{n}\right)$ isotopic to $\Omega$, with piecewise smooth boundary, which contains all the conical singularities corresponding to the faces of $\Gamma$ contained in $\Omega$, but no other conical singularity. Let $k_{n}$ be the integral of the geodesic curvature of $\partial \Omega_{n}$, the Gauss-Bonnet theorem shows that

$$
k_{n}=2 \pi \chi(\Omega)-\sum_{f \subset \Omega} \kappa_{n}(f)-K_{n} A\left(\Omega_{n}\right) .
$$

We can choose the $\Omega_{n}, n \in \mathbb{N}$, so that $\left(\Omega_{n}\right)_{n \in \mathbb{N}}$ converges, as $n \rightarrow \infty$, to a limit domain $\Omega_{\infty}$ in the flat surface with boundary and with one singular point which we already mentioned above. Let $k$ be the integral of the geodesic curvature of $\partial \Omega_{\infty}$. Then, taking the limit in the previous equation

$$
k=2 \pi \chi(\Omega)-\sum_{f \subset \Omega} \kappa(f)-K_{\infty} A\left(\Omega_{\infty}\right) .
$$

Let $k_{1}, \ldots, k_{N}$ the singular curvatures at $x_{i}$ of the flat surface $S_{i}, 1 \leq i \leq N$. Then, for each of those surfaces, the integral of the geodesic curvature of the boundary of any domain containing the singular point is $2 \pi-k_{i}$ minus $K_{\infty}$ times the area of the corresponding disk, by the Gauss-Bonnet Theorem. It follows that

$$
\sum_{i=1}^{N}\left(2 \pi-k_{i}\right)=2 \pi \chi(\Omega)-\sum_{f \subset \Omega} \kappa(f) .
$$

To each $\partial_{i} \Omega, 1 \leq i \leq N$ we associate a sequence of circles containing the cone point $x_{i}$, namely the circles corresponding to the vertices of $\partial_{i} \Gamma_{1}^{s}$. Lemma 3.1, applied to those circles, shows that the sum of their intersection angles in the limit is equal to $2 \pi-k_{i}$. But their intersection angles are of two types:

- each dual circle intersects orthogonally two principal circles, so that the total contribution of each dual circle is equal to $\pi$, and 
- each edge of $\partial_{i} \Gamma_{1}^{S}$, with endpoints corresponding to two principal circles, corresponds to an edge of the connected component of $\partial \Omega$ corresponding to $\gamma_{i}$.

Consider (7), replacing the left-hand side by the sum of the intersection angles between the circles, we obtain

$$
\sum_{e \in \partial \Omega} \theta(e)+m(\Omega) \pi=2 \pi \chi(\Omega)-\sum_{f \subset \Omega} \kappa(f),
$$

which means precisely that the inequality in condition (2) of Theorem 1.4 or Theorem 1.6 is an equality. Note also that $\Gamma_{1}^{s}$ contains at least one vertex corresponding to a principal circle, and it follows that $\Omega$ is not a face of $\Gamma$. So the equality above is a contradiction.

\subsection{Proof of the Compactness Lemma}

Proposition 6.3, along with its hyperbolic analog, is the main tool for the proof of Lemma 6.1. Once it is known, the compactness results are obtained by using elementary geometric arguments concerning the constant curvature metrics on the surfaces with boundary obtained by considering a finite (but increasing) set of overlapping disks.

Consider a graph $\Gamma$ and some numbers $w_{i} \in(0, \pi)$ associated to the edges of $\Gamma$. A hyperideal circle pattern with incidence graph $\Gamma$ and intersection angles given by the $w_{i}$, and its underlying constant curvature $K$ metric, are determined by a finite set of data:

- the curvature $K$,

- the radii of the principal circles,

- the singular curvatures at the centers of the dual circles, and

- an "angle" data describing the relative positions of the circles intersecting a given principal circle, say $C_{i}$ : if $C_{j}$ and $C_{k}$ intersect $C_{i}$, it is the angle under which the centers of $C_{j}$ and $C_{k}$ are "seen" from the center of $C_{i}$.

Indeed given this information the radii and position of the dual circles follow, and it is not difficult to check that this completely determines the underlying metric and circle pattern.

Proposition 6.3 shows that the radii of the principal circles remain bounded, and the singular curvatures at the centers of the dual circles converge. The angle data are defined in a compact set. Therefore after taking a subsequence all those data converge. Since the radii of the principal circles remain bounded from below, the injectivity radii of the underlying sequence of metrics remain bounded from below.

Notice that the combinatorics of the circle pattern remain the same in the limit. Clearly two circles corresponding to adjacent vertices of $\Gamma$ remain so in the limit, because they still intersect and their intersection points remain in interstices. Conversely, two circles not adjacent cannot become adjacent in the limit, which would mean that either they become tangent and their tangency point is in an interstice, or that their intersection points are contained, in the limit, in interstices. This would be forbidden by the constraints on a hyperideal circle pattern. For instance if $C_{1}$ and $C_{2}$ are two principal circles which are not adjacent in the sequence, but which are both 
orthogonal to a dual circle $C^{\prime}$ then, if $C_{1}$ and $C_{2}$ where tangent in the limit, it would imply that (still in the limit) $C^{\prime}$ would have to be on either side of $C_{1} \cup C_{2}$, and that it could not be adjacent to some of the principal circles which corresponds to vertices of $\Gamma$ contained in the face corresponding to $C^{\prime}$ "between" the vertices corresponding to $C_{1}$ and $C_{2}$.

Moreover, the distance between the centers of the dual circles is also bounded from below because, as is not difficult to see, the centers of two dual circles can "collapse" only if the radii of those circles go to 0 and the intersection between two principal circles, both adjacent to those two dual circles, goes to $\pi$. It follows that the limit metric is a constant curvature metric with conical singularities and the corresponding circle pattern has combinatorics given by $\Gamma$ and intersection angles and singular curvatures prescribed by $\theta$ and $\kappa$.

\section{Proofs of the Main Results on Closed Surfaces}

\subsection{Outline}

The previous section contains results showing that the map sending a hyperideal circle pattern on a singular surface to its intersection angles and the singular curvatures at the singular points is a local homeomorphism, and also that it is proper. It follows that it is a covering of the spaces of possible intersection angles and singular curvatures - in the different contexts considered here-by the corresponding spaces of hyperideal circle patterns. However, it remains necessary to prove that the number of inverse images of each set of intersection angles and singular curvatures, which is locally constant, is equal to 1 .

Two different arguments will be used. For the uniqueness- the fact that each set of intersection angles and singular curvatures has at most one inverse image-we use the fact that circle patterns correspond to critical points of the volume over polytopes, while the volume is a strictly concave function. This is done in Sect. 7.2.

It is also necessary to prove that, for any given combinatorics, there are some assignments of intersection angles and singular curvatures which are indeed realized by a hyperideal circle pattern. This is done first for hyperbolic surfaces in Sect. 7.3, using the Orbifold Hyperbolization Theorem (see [6]) and a double doubling argument, which shows that, to some strictly hyperideal circle pattern on a hyperbolic surface, it is possible to associate a hyperbolic orbifold, and conversely.

Then, in Sect. 7.4, we use the existence result for some hyperbolic circle patterns to obtain a similar existence result for some Euclidean circle patterns, using an approximation by hyperbolic circle patterns and Lemma 6.1.

\subsection{Uniqueness}

The proof of the uniqueness of circle patterns with a given combinatorics, intersection angles and singular curvatures, follows quite directly from the constructions made in Sect. 5 to prove the infinitesimal rigidity. 
Closed Hyperbolic Surfaces Consider first the case of circle patterns with incidence graph $\Gamma$ on a closed surface $\Sigma$ of genus at least 2 with a singular hyperbolic metric. The fact that $\mathcal{D}(\Gamma)$ is connected follows from its definition, which shows that it is actually a polytope.

We have seen in Sect. 5 the definition of a polytope $\mathcal{A}_{H}(\Sigma, \Gamma)$, on which two functions are defined:

- the volume function, $\mathcal{V}$, which is strictly concave, and

- the affine function $\sigma: \mathcal{A}_{H}(\Sigma, \Gamma) \rightarrow \mathcal{D}(\Sigma, \Gamma)$.

Moreover, the circle patterns are associated to the critical points of the restriction of $\mathcal{V}$ to the level sets of $\sigma$ (see Lemmas 5.5 and 5.6). At each such critical point, the value of $\sigma$ determines the intersection angles and the singular curvatures of the circle pattern. Since $\sigma$ is affine, its level sets are affine subsets of $\mathcal{A}_{H}(\Sigma, \Gamma)$. Moreover, the restriction of $\mathcal{V}$ to those level sets remains strictly concave, so that $\mathcal{V}$ has at most one critical point on each level set. This shows that each family of intersection angles and singular curvatures is obtained on at most one circle pattern, proving the uniqueness in Theorem 1.6.

Euclidean Surfaces The same argument can be used for Euclidean circle patterns on closed surfaces, i.e. Theorem 1.4. The relevant polytope is now $\mathcal{A}_{E}(\Sigma, \Gamma)$ (see Definition 5.1), but it remains true that circle patterns are in one-to-one correspondence with the critical points of the restriction of $\mathcal{V}$ to the level sets of $\sigma$ (see Lemma 5.9). The uniqueness of the circle patterns in Theorem 1.4 thus follows.

\subsection{Existence for Hyperbolic Metrics}

The Orbifold Hyperbolization Theorem We will use an important statement, discovered by Thurston (who gave an outline of the proof) and which has recently been extensively proved $[5,6,13]$.

Theorem 7.1 (Thurston, see [5, 6]) Let $M$ be a compact, connected, orientable, irreducible three-dimensional orbifold with nonempty singular locus. If $M$ is topologically atoroidal, then $M$ is geometric.

It will be applied here for Haken orbifolds, for which a simpler proof has been available for some time.

To check that an orbifold, with singular locus a disjoint union of closed curves, has a hyperbolic orbifold structure, it is sufficient (cf. [5]) to check that:

- every essential sphere intersects singular lines on which the sum of the singular curvatures is at least $4 \pi$, and

- every essential torus intersects at least one singular line.

This statement will be applied to a three-dimensional orbifold constructed by simple doubling constructions from a hyperbolic circle pattern. 
Doubling Constructions We first consider a hyperideal circle pattern $C$ on a hyperbolic surface $\Sigma$, with incidence graph $\Gamma$, singular curvatures given by $\kappa: \Gamma_{2} \rightarrow$ $(-\infty, 2 \pi)$, and angle intersections given by $\theta: \Gamma_{1} \rightarrow(0, \pi)$. We suppose that:

- no interstice of $C$ is reduced to a point,

- for each $f \in \Gamma_{2}, \kappa(f)=2 \pi-2 \pi / k$, for some integer $k \geq 2$, and

- for each $e \in \Gamma_{1}, \theta(e)=\pi-\pi / k$, for some integer $k \geq 2$.

We have seen in Sect. 4 how to construct from $C$ a cellular complex $\mathcal{S}$, and a complete hyperbolic metric with cone singularities $H(C)$ on $\mathcal{S}$. Moreover, each of the three-dimensional cells of $\mathcal{S}$ has one face corresponding to a face of $\Gamma^{*}$, and another corresponding to a vertex $v_{0}$ which is the same for all three-cells of $\mathcal{S}$. In the hyperbolic metric $H(C), v_{0}$ corresponds to a strictly hyperideal vertex-because the circle pattern $C$ is on a hyperbolic surface-while all other vertices correspond to strictly hyperideal vertices-because we have supposed that the interstices of $C$ are "strictly hyperideal" (they are not reduced to points).

We define another cellular complex $\mathcal{S}_{t}$ with a hyperbolic cone-metric $H_{t}(C)$ from $(\mathcal{S}, H(C))$ by truncating it at the dual planes of all its vertices (which are all strictly hyperideal). The resulting cone-manifold is compact, homeomorphic to the product of $\Sigma$ by an interval, with a boundary made of three different parts:

- $\partial_{0} \mathcal{S}_{t}$, defined as the union of the faces dual to $v_{0}$ in all the simplices of $\mathcal{S}$. It follows from the construction in Sect. 4 that $\partial_{0} \mathcal{S}_{t}$ is connected and totally geodesic.

- $\partial_{t} \mathcal{S}_{t}$, the union of the faces dual to all the vertices of $\mathcal{S}$ other than $v_{0}$. Also by construction, $\partial_{t} \mathcal{S}_{t}$ is a disjoint union of totally geodesic polygons, each corresponding to one of the vertices of $\mathcal{S}$ other than $v_{0}$.

- $\partial_{p} \mathcal{S}_{t}$, which is the part of $\partial \mathcal{S}_{t}$ which was already contained in $\partial \mathcal{S} . \partial_{p} \mathcal{S}_{t}$ is topologically $\Sigma$ with one disk removed for each face of $\Gamma$.

In addition, $\mathcal{S}_{t}$ has one singular line for each face $f$ of $\Gamma$, with total angle equal to $\kappa(f) . \partial_{p} \mathcal{S}_{t}$ is polyhedral, it has one edge for each edge $e$ of $\Gamma$, with exterior dihedral angle equal to $\theta(e)$.

We now define a second simplicial complex $\mathcal{S}_{2}$ with a hyperbolic cone-metric $H_{2}(C)$ by gluing two copies of $\left(\mathcal{S}_{t}, H_{t}(C)\right)$ isometrically along $\partial_{0} \mathcal{S}_{t}$ and $\partial_{t} \mathcal{S}_{t}$. The resulting cone-manifold is topologically the product of $\Sigma$ by an interval with, for each face of $\Gamma$, one handle connecting one face with the other. It also has one singularity along a closed line for each face $f$ of $\Gamma$, with total angle $2 \pi-\kappa(f)$. Its boundary is "polyhedral", with one face corresponding to each vertex of $\Gamma$, one edge-which is a closed curve-for each edge of $\Gamma$, and no vertex.

Finally we define a third cone-manifold, $\mathcal{S}_{4}$, with a hyperbolic cone-metric $H_{4}(C)$, by gluing two copies of $\left(\mathcal{S}_{2}, H_{2}(C)\right)$ isometrically along their boundary. By construction, the total angle around each singular line-which corresponds either to a face or to an edge of $\Gamma$-is equal to $2 \pi / k$, for some $k \geq 2$. Therefore, $\left(\mathcal{S}_{4}, H_{4}(C)\right)$ is a hyperbolic orbifold.

Construction of Hyperbolic Orbifolds We now consider the same constructions in a topological manner, with no hyperbolic metric involved. Consider a graph $\Gamma$ embedded in $\Sigma$ and two functions $\kappa: \Gamma_{2} \rightarrow(0,2 \pi)$ and $\theta: \Gamma_{1} \rightarrow(0, \pi)$, satisfying the hypothesis of Theorem 1.6, such that $\kappa(f)=2 \pi(1-1 / k)$ for some $k \geq 2$ and that 
$\theta(e)=\pi-\pi / k$ for some $k \geq 2$, for all face $f$ (resp. for all edge $e$ ) of $\Gamma$. By the same construction as above, it is possible to construct a cone-manifold $\mathcal{S}_{4}$, with singularities along closed curves, with angle $2 \pi / k$ (for some $k \geq 2$ ) along each of those curves, so $\mathcal{S}_{4}$ is an orbifold.

\section{Lemma 7.2 $\mathcal{S}_{4}$ admits a hyperbolic orbifold structure.}

Proof $\mathcal{S}_{4}$ was constructed above by gluing along their boundary two copies of $\mathcal{S}_{2}$, which we call $\mathcal{S}_{2,+}$ and $\mathcal{S}_{2,-}$. Each is topologically the product of $\Sigma$ by an interval, with one handle for each face of $\Gamma$. The boundary of $\mathcal{S}_{2,+}$ and $\mathcal{S}_{2,-}$ contain some singular curves of $\mathcal{S}_{4}$, those corresponding to an edge of $\Gamma$. To each such curve is associated an "exterior angle", which is half the singular curvature of the corresponding curve in $\mathcal{S}_{4}$.

Consider an essential torus $T^{2} \subset \mathcal{S}_{4}$. Suppose first that $\Sigma$ is not a torus. Given the topology of $\mathcal{S}_{2,+}$ and $\mathcal{S}_{2,-}$, they do not contain any essential torus, so the intersection of $T^{2}$ with $\mathcal{S}_{2,+}$ (resp. $\mathcal{S}_{2,-}$ ) contains an essential annulus $A_{+}$(resp., $A_{-}$). The boundary of $A_{+}$is made of two curves on $\partial \mathcal{S}_{2}$, each of which intersects at least one curve corresponding to an edge of $\Gamma$. So, in $\mathcal{S}_{4}, T^{2}$ intersects at least one of the singular curves of $\mathcal{S}_{4}$.

If $\Sigma$ is a torus, the same argument holds except in the case where $T^{2}$ is contained in $\mathcal{S}_{2_{+}}$(or in $\mathcal{S}_{2,-}$ ); then it has to intersect at least one of the singular curves corresponding to the faces of $\Gamma$.

Now let $S$ be an essential sphere in $\mathcal{S}_{4}$. Since $\mathcal{S}_{2,+}$ and $\mathcal{S}_{2,-}$ are irreducible, the intersection of $S$ with $\mathcal{S}_{2,+}$ (resp., $\mathcal{S}_{2,-}$ ) contains at least one essential disk $D_{+}$(resp., $\left.D_{-}\right)$. The boundary of $D_{+}$is a closed curve in $\partial \mathcal{S}_{2,+}$. Let $K_{i}\left(D_{+}\right)$be the sum of the singular curvatures associated to the singular curves in $\mathcal{S}_{2,+}$ which intersect the interior of $D_{+}$, and let $\Theta_{b}\left(D_{+}\right)$be the sum of the "exterior angles" associated to the curves in $\partial \mathcal{S}_{2,+}$ (corresponding to the edges of $\Gamma$ ) which intersect $\partial D_{+}$. We will show that

$$
K_{i}\left(D_{+}\right)+\Theta_{b}\left(D_{+}\right)>2 \pi .
$$

$\mathcal{S}_{2,+}$ is itself obtained by gluing two copies of $\mathcal{S}_{t}$, which we call $\mathcal{S}_{t,+}$ and $\mathcal{S}_{t,-}$, along one boundary component and along disks corresponding to the faces of $\Gamma$. Let $D_{+,+}:=D_{+} \cap \mathcal{S}_{t,+}, D_{+,-}:=D_{+} \cap \mathcal{S}_{t,-}$. After deformation of $D$, we can suppose that $D_{+,+}$and $D_{+,-}$have a minimal number of connected components. We consider three cases.

1. $D_{+,-}=\emptyset$. Consider the projection of $D_{+,+}$on $\partial \mathcal{S}_{t,+}$, it is a disk with boundary equal to $\partial D_{+,+}$(which does not intersect the disks in $\partial \mathcal{S}_{t,+}$ corresponding to the faces of $\Gamma$ ). Consider the sequence of edges of $\Gamma^{*}$ which intersect $\partial D_{+,+}$, the corresponding edges of $\Gamma$ are the boundary of an admissible domain $\Omega$ in $(\Sigma, \Gamma)$, which is topologically a disk, with $m(\Omega)=0$. Condition (2) in Theorem 1.6 is that:

$$
\sum_{e \in \Gamma_{1}, e \subset \partial \Omega} \theta(e) \geq(2 \chi(\Omega)-m(\Omega)) \pi-\sum_{f \in \Gamma_{2}, f \subset \Omega} \kappa(f)=2 \pi-\sum_{f \in \Gamma_{2}, f \subset \Omega} \kappa(f),
$$

which means precisely that $\Theta_{b}\left(D_{+}\right)>2 \pi-K_{i}\left(D\left(_{+}\right)\right.$, as needed. 
2. $D_{+,+}=\emptyset$. The same argument can then be used with $D_{+,-}$instead of $D_{+,+}$.

3. $D_{+,-}$and $D_{+,+}$are both nonempty. There are then at least one connected component of $D_{+,+}$, called $D_{+,+}^{\prime}$, with exactly one boundary component contained in one of the disks, corresponding to a face $f$ of $\Gamma$, along which $\mathcal{S}_{t,+}$ is glued to $\mathcal{S}_{t,-}$. Considering as before the sequence of edges of $\Gamma$ corresponding to the edges of $\Gamma^{*}$ intersected by $\partial D_{+,+}^{\prime}$, and adding a segment in $f$, we obtain an admissible domain $\Omega_{+,+}$in $(\Sigma, \Gamma)$, which is a disk with $m\left(\Omega_{+,+}\right)=1$. Condition (2) of Theorem 1.6 now shows that the sum of the exterior angles associated to the edges intersected by $\partial D_{+,+}^{\prime}$ is larger than $\pi$ minus the sum of the singular curvatures associated to the singular curves of $\mathcal{S}_{2,+}$ intersected by $D_{+,+}^{\prime}$. The same argument can be use for a connected component $D_{+,-}^{\prime}$ of $D_{+,-}$with exactly one boundary component contained in one of the disks on $\partial \mathcal{S}_{t,-}$ corresponding to a face of $\Gamma$, and the sum of the two contributions shows (8).

The same argument can also be applied to $D_{-}$, and it shows that, using the same notations for $D_{-}$as for $D_{+}: K_{i}\left(D_{-}\right)+\Theta_{b}\left(D_{-}\right)>2 \pi$, while by definition $\Theta_{b}\left(D_{-}\right)=$ $\Theta_{b}\left(D_{+}\right)$. However, the sum of the singular curvatures of the singular curves which intersect $S$ is equal to $K_{i}\left(D_{+}\right)+K_{i}\left(D_{-}\right)+2 \Theta_{d}\left(D_{+}\right)$, so it is larger than $4 \pi$. This means that each essential sphere in $\mathcal{S}_{4}$ intersects singular curves with a sum of singular curvatures larger than $4 \pi$.

It now follows from the Orbifold Hyperbolization Theorem that $\mathcal{S}_{4}$ has a hyperbolic orbifold structure.

Notice that condition (2) of Theorem 1.6 is used here only in the case where $\Omega$ is a disk. This is quite normal since we consider here circle patterns on hyperbolic surfaces with positive singular curvature at the cone points, and we have already seen in Sect. 1 that, in that case, condition (2) is void except when $\Omega$ is a disk.

An Existence Result in Some Special Cases Let $\Gamma$ be the one-skeleton of a cellular decomposition of a closed surface $\Sigma$. We have seen earlier that, from a circle pattern of incidence graph $\Gamma$, with intersection angles and singular curvatures satisfying some simple conditions, it is possible to "build" a hyperbolic orbifold structure on $\mathcal{S}_{4}$. The argument given right above using the Orbifold Hyperbolization Theorem shows that, conversely, given $\Gamma$ and two functions $\theta$ and $\kappa$ satisfying the same conditions, there is a unique hyperbolic orbifold structure on $\mathcal{S}_{4}$ with total angles around the singular curves given by $2 \pi-\kappa$ and by $2(\pi-\theta)$ on singular curves corresponding to faces or to edges of $\Gamma$, respectively. By uniqueness, this metric has the same symmetry as $\mathcal{S}_{4}$, so it can be cut along a polyhedral surface to obtain two hyperbolic cone-manifolds with boundary, $\mathcal{S}_{2,+}$ and $\mathcal{S}_{2,-}$, which are isometric.

Then, again by uniqueness, it is possible to "cut" $\mathcal{S}_{2,+}$ along a totally geodesic surface $\Sigma_{0}$ and a disjoint union of disks, to obtain a cone-manifold $\mathcal{S}_{t}$. The boundary of $\mathcal{S}_{t}$ has two connected component, one corresponding to $\Sigma_{0}$ which is totally geodesic, and another one which is polyhedral, with some faces corresponding to the faces of $\Gamma$ and other corresponding to the vertices of $\Gamma$. Moreover two faces corresponding to the endpoints of an edge $e$ of $\Gamma$ are adjacent, and the exterior dihedral angle between them is $\theta(e)$. It is possible to project orthogonally on $\Sigma_{0}$ the boundaries at infinity of the hyperbolic planes containing the faces of $\left(\partial \mathcal{S}_{2,+}\right) \backslash \Sigma_{0}$ corresponding to vertices 
of $\Gamma$, one obtains in this manner a hyperideal circle pattern on $\Sigma_{0}$, with incidence graph $\Gamma$, singular curvatures given by $\kappa$, and intersection angles given by $\theta$.

This result can be stated as follows.

Lemma 7.3 Let $\Sigma$ be a closed surface, let $\Gamma$ be the one-skeleton of a cellular decomposition of $\Sigma$, and let $\kappa: \Gamma_{2} \rightarrow(0,2 \pi)$ and $\theta: \Gamma_{1} \rightarrow(0, \pi)$ be two functions such that:

- $\Gamma, \kappa$ and $\theta$ satisfy the hypothesis of Theorem 1.6;

- the sum of the values of $\theta$ on the boundary of each face $f$ of $\Gamma$ is strictly larger than $2 \pi-\kappa(f)$;

- for each $f \in \Gamma_{2}, \kappa(f)=2 \pi-2 \pi / k$, for some integer $k \geq 2$; and

- for each $e \in \Gamma_{1}, \theta(e)=\pi-\pi / k$, for some integer $k \geq 2$.

Then there is a strictly hyperideal circle pattern $C$ on $\Sigma$, with incidence graph $\Gamma$, singular curvatures given by $\kappa$, and intersection angles given by $\theta$.

Proof of Theorem 1.5 Let again $\Sigma$ be a closed surface, and let $\Gamma$ be the one-skeleton of a cellular decomposition of $\Sigma$. Following the notation from Sect. 2, we call $\mathcal{D}(\Gamma)$ the vector space of functions $\kappa, \theta$ satisfying the hypothesis of Theorem 1.6.

Notice that, when $\Sigma$ is a sphere, $\Gamma$ needs to have at least three faces for condition (1) of Theorem 1.6 to apply.

Lemma 7.4 There exist functions $\kappa, \theta$ which are in $\mathcal{D}(\Gamma)$ and for which:

1. the sum of the values of $\theta$ on the boundary of each face $f$ of $\Gamma$ is strictly larger than $2 \pi-\kappa(f)$;

2. for each face $f \in \Gamma_{2}, \kappa(f)=2 \pi-2 \pi / k$, for some integer $k \geq 2$; and

3. for each $e \in \Gamma_{1}, \theta(e)=\pi-\pi / k$, for some integer $k \geq 2$.

Proof Let $k \in \mathbb{N}, k \geq 2$. Choose $\kappa(f)=2 \pi(1-1 / k)$ for all $f \in \Gamma_{2}$, and let $\theta(e)=$ $\pi(1-1 / k)$ for all $e \in \Gamma_{1}$. For each face $f$ of $\Gamma, f$ has at least three edges, so the sum of the values of $\theta$ on the boundary of $f$ is at least equal to $3 \pi-3 \pi / k$. On the other hand, $2 \pi-\kappa(f)=2 \pi / k$. Since $k \geq 2$, condition (1) above is always satisfied.

We now have to prove that, if $k$ is chosen large enough, $\theta$ and $\kappa$ correspond to a point in $\mathcal{D}(\Gamma)$, i.e. that the conditions in Theorem 1.6 are satisfied. Condition (1) is simply that the sum of $\kappa(f)$ over the faces of $f$ is larger than $\chi(\Sigma)$; since $\kappa(f)>0$ for all $f \in \Gamma_{2}$, this is clearly true when $\Sigma$ is a torus or a higher genus surface. When $\Sigma$ is a sphere, $\Gamma$ has at least three faces, so the condition is satisfied as soon as $k \geq 4$.

Since $\kappa(f)>0$ for all faces of $\Gamma$, the right-hand side of the second condition in Theorem 1.6 is negative unless $\Omega$ is a disk with $m(\Omega) \in\{0,1\}$. Moreover, if $k \geq 4$, it will also be negative as soon as $\Omega$ contains two faces of $\Gamma$, since $2 \pi-2 \times 2 \pi$ (1$1 / k)<0$, and also if $\Omega$ contains one face of $\Gamma$ and $m(\Omega)=1$. So the only case left is when $\Omega$ is a face of $\Gamma$ and $m(\Omega)=0$, and then, since each face has at least 3 edges, the condition applies if $k \geq 4$.

The proof of Theorem 1.6 is now clear. Given $\Sigma$ and $\Gamma$, we consider the map $\Phi$ on $\mathcal{C}(\Gamma)$ sending a hyperideal circle pattern to the data given by its intersection angles 
and singular curvatures. We have seen in Sect. 3 that $\Phi_{\Gamma}$ takes its values in $\mathcal{D}(\Gamma)$. Moreover Lemma 5.7 shows that $\Phi_{\Gamma}$ is a local homeomorphism, and Lemma 6.1 shows that it is proper, so that $\Phi_{\Gamma}$ is a covering of $\mathcal{D}(\Gamma)$ by $\mathcal{C}(\Gamma)$. It was also proved in Sect. 7.1 that the number of inverse images of each point in $\mathcal{D}(\Gamma)$ is at most equal to 1 . But Lemma 7.4 shows that $\mathcal{D}(\Gamma)$ contains at least one "special" point which, by Lemma 7.3, has one inverse image. So $\Phi_{\Gamma}$ is a homeomorphism.

\subsection{Euclidean Metrics}

Outline of the Proof The proof of Theorem 1.4 follows the same path as the proof of Theorem 1.6 except for the last step. As for Theorem 1.6, given a closed surface $\Sigma$ and a graph $\Gamma$ embedded in $\Sigma$ which is the one-skeleton of a cellular decomposition, we can consider the space $\mathcal{C}(\Gamma)$ of hyperideal circle patterns on $\Sigma$ with incidence graph $\Gamma$, and the map $\Phi_{\Gamma}$ sending a hyperideal circle pattern to its singular curvatures - a function defined on the faces of $\Gamma$ - and its intersection angles - a function defined on the edges of $\Gamma$. It follows from Sect. 3 that the image of $\Phi_{\Gamma}$ is contained in $\mathcal{D}(\Gamma)$, the set of curvature and intersection data allowed by Theorem 1.4, while Lemma 5.10 shows that $\Phi_{\Gamma}$ is a local homeomorphism, and Lemma 6.1 shows that $\Phi_{\Gamma}$ is proper. The arguments given above in the hyperbolic case can also be used to show that each element of $\mathcal{D}(\Gamma)$ has at most one inverse image by $\Phi_{\Gamma}$.

The only remaining point is that, for all $\Gamma$, any element of $\mathcal{D}(\Gamma)$ has at least one inverse image by $\Phi_{\Gamma}$. This will be proved using Theorem 1.6 and the compactness stated in Lemma 6.1 .

An Approximation Argument Let $\kappa: \Gamma_{2} \rightarrow(-\infty, 2 \pi)$ and $\theta: \Gamma_{1} \rightarrow(0,2 \pi)$ describe an element of $\mathcal{D}(\Gamma)$, i.e. $\kappa$ and $\theta$ satisfy the hypothesis of Theorem 1.4.

Lemma 7.5 There exists sequences $\left(\kappa_{n}\right)_{n \in \mathbb{N}}$ and $\left(\theta_{n}\right)_{n \in \mathbb{N}}$, with $\kappa_{n}: \Gamma_{2} \rightarrow(-\infty, 2 \pi)$ and $\theta_{n}: \Gamma_{1} \rightarrow(0, \pi)$, defined for $n \geq n_{0}$ for some $n_{0} \in \mathbb{N}$, such that:

- for all $n \geq n_{0}, \kappa_{n}$ and $\theta_{n}$ satisfy the hypothesis of Theorem 1.6, and therefore are the singular curvature and intersection angles of a unique hyperideal circle pattern on $\Sigma$ with a hyperbolic metric; and

- for all $f \in \Gamma_{2}$ and all $e \in \Gamma_{1}, \kappa_{n}(f) \rightarrow \kappa(f)$ and $\theta_{n}(e) \rightarrow \theta(e)$ as $n \rightarrow \infty$.

Proof Let $\kappa_{n}$ and $\theta_{n}$ be defined by

$$
\forall f \in \Gamma_{2}, \quad \kappa_{n}(f)=\kappa(f)+\frac{1}{n}, \quad \forall e \in \Gamma_{1}, \quad \theta_{n}(e)=\theta(e)+\frac{1}{n} .
$$

We have supposed that $\kappa$ and $\theta$ satisfy the hypothesis of Theorem 1.4, in particular the sum of the $\kappa(f)$ over all faces of $\Gamma$ is equal to $2 \pi \chi(\Sigma)$. So, for all $n$, the sum of $\kappa_{n}(f)$ over all faces of $\Gamma$ is strictly larger than $2 \pi \chi(\Sigma)$, so that condition (1) of Theorem 1.6 holds.

Let $\Omega$ be an admissible domain in $(\Sigma, \Gamma)$. Still by our hypothesis, the second condition of Theorem 1.4 holds for $\Omega$ :

$$
\sum_{e \in \Gamma_{1}, e \subset \partial \Omega} \theta(e) \geq(2 \chi(\Omega)-m(\Omega)) \pi-\sum_{f \in \Gamma_{2}, f \subset \Omega} \kappa(f),
$$


with strict inequality except perhaps when $\Omega$ is a face of $\Gamma$. If $\Omega$ is a face of $\Gamma$, then it has at least three edges, and clearly this inequality is also valid for $\kappa_{n}$ and $\theta_{n}$, for any $n \geq 1$. Otherwise, the inequality is strict, and it follows that, for $n$ large enough (depending on $\Omega$ ) the inequality holds for $\kappa_{n}$ and $\theta_{n}$. Since there is a finite number of admissible domains in $(\Sigma, \Gamma)$, it follows that for $n$ large enough the hypothesis of Theorem 1.6 hold.

Proof of Theorem 1.4 The proof of Theorem 1.4 now follows from a direct application of Lemma 6.1 to the hyperideal circle patterns associated by Theorem 1.6 to the functions $\kappa_{n}$ and $\theta_{n}$, which are on hyperbolic surfaces which, after applying a sequence of homotheties so that their area remains equal to 1, converge to Euclidean surfaces.

\section{End of the Proofs, Remarks}

The first part of this section contains the proof of the main results concerning circle patterns on surfaces with polygonal boundary, and the next contains a remark and some open questions.

\subsection{Circle Patterns on Surfaces with Boundary}

We turn here to the proof of Theorem 1.10 and Theorem 1.11. The proofs of those two results follow the same pattern, so we give the arguments mainly for Theorem 1.11 only, leaving the (limited) adaptation necessary for the Euclidean context to the interested reader. We consider now a hyperbolic surface $\Sigma$ with polygonal boundary, along with a circle pattern $C$-as in the statement of Theorem 1.11 -with incidence graph $\Gamma$ and extended incidence graph $\Gamma^{\prime}$.

Doubling a Circle Pattern We consider a closed surface with conical singularities, called $D(\Sigma)$, which is the "double" of $\Sigma$; it is obtained by gluing two copies of $\Sigma$ isometrically along their common boundary, the gluing being made through the identity map. There is also a circle pattern in $D(\Sigma)$, which we call $D(C)$, which is the inverse image of $C$ under the canonical projection from $D(\Sigma)$ to $\Sigma$, so that each circle of $C$ lifts to the two circles in $D(\Sigma)$, corresponding to $C$ in the two copies of $\Sigma$.

It is then a simple matter to check that the incidence graph of $D(C)$ is a graph embedded in $D(\Sigma)$, which we call $D(\Gamma)$ here, which has:

- two vertices for each vertex of $\Gamma$, i.e. for each vertex of $\Gamma^{\prime}$ which is not on the boundary of the exterior face;

- two faces for each face of $\Gamma$, and one for each face of $\Gamma^{\prime}$ which does not correspond to a face of $\Gamma$; and

- two edges for each edge of $\Gamma$, and one edge for each edge of $\Gamma^{\prime}$ which is going from an "interior" vertex of $\Gamma^{\prime}$ to a "boundary" vertex of $\Gamma^{\prime}$.

Moreover, the angle assigned to an edge of $D(\Gamma)$ is: 
- for edges corresponding to edges of $\Gamma$, the intersection angle between the two circles of $C$ corresponding to its endpoints; and

- for edges corresponding to edges of $\Gamma^{\prime}$ going from an "interior" to a "boundary" vertex, twice the angle on that edge (since the intersection angle between the circles corresponding to the endpoints is twice the angle between one of those circles and the corresponding boundary segment).

Finally, the singular curvature assigned to a face of $D(\Gamma)$ is:

- for faces corresponding to faces of $\Gamma$, identical to the singular curvature of that face; and

- for faces corresponding to faces of $\Gamma^{\prime}$ which are not faces of $\Gamma$, twice the angle assigned to the exterior edge of that face in $\Gamma^{\prime}$, since the singular angle of $D(\Sigma)$ at that point is twice the exterior angle of $\partial \Sigma$ at the corresponding point.

This defines assignments $D(\kappa)$ and $D(\theta)$ of angles to the faces and edges of $D(\Gamma)$, respectively. Note that there is a slight abuse of notation here since $D(\kappa)$ depends not only on $\kappa$ but also on $\theta$ (because the values of $D(\kappa)$ on faces corresponding to faces of $\Gamma^{\prime}$ which are not faces of $\Gamma$ depend on the values of $\theta$ on the exterior edges of those faces).

The key point of the proofs is then:

Lemma 8.1 $\Gamma, \kappa$ and $\theta$ satisfy the conditions of Theorem 1.11 if and only if $D(\Gamma)$, $D(\kappa)$ and $D(\theta)$ satisfy the condition of Theorem 1.6.

Proof First note that condition (1) of Theorem 1.6 for the doubled surface is equivalent to condition (1) of Theorem 1.11 for the original surface with boundary, because the curvature associated to each face of $\Gamma$ counts once in each of the copies of $\Sigma$ which are glued, the curvatures at the vertices of the gluing line are equal to twice the exterior angles at the corresponding vertices of $\partial \Sigma$, while $\chi(D(\Sigma))=2 \chi(\Sigma)$.

Suppose that $D(\Gamma), D(\kappa)$ and $D(\theta)$ satisfy the condition of Theorem 1.6. Let $\Omega$ be an admissible domain in $\left(\Sigma, \Gamma^{\prime}\right)$, and let $D(\Omega)$ be the domain in $D(\Sigma)$ which is the inverse image of $\Omega$ under the canonical projection from $D(\Sigma)$ to $\Sigma$. We already know that (1) holds for $D(\Omega)$, considered as an admissible domain in $(D(\Sigma), D(\Gamma))$, so that

$$
\sum_{\substack{e \in D(\Gamma)_{1}, e \subset \partial D(\Omega)}} \theta(e)=\pi(2 \chi(D(\Omega))-m(D(\Omega)))-\sum_{\substack{f \in D(\Gamma)_{2}, f \subset D(\Omega)}} \kappa(f) .
$$

However, the description of $D(\Gamma)$ - and of the corresponding angles and singular curvatures-shows that

$$
\sum_{e \subset \partial D(\Omega)} \theta(e)=2 \sum_{e \in \Gamma_{1}} \theta(e)+\sum_{\substack{e \in \Gamma_{1}^{\prime} \backslash \Gamma_{1}, e \subset \partial \Omega \backslash \partial \Sigma}} 2 \theta(e)
$$

where the first sum is over the edges of $\partial \Omega$ which are in $\Gamma$, while the second sum is over the edges of $\Gamma^{\prime}$ which have one vertex on the exterior face of $\Gamma^{\prime}$. The coefficient 2 in the first sum comes from the fact that each edge of $\Gamma$ corresponds to two edges 
in $D(\Gamma)$, while the same coefficient in the second sum comes from the fact that the angle between the two circles corresponding to the endpoints in $D(C)$ is twice the angle between the corresponding circle of $C$ and the adjacent segment of $\partial \Sigma$. In the same way

$$
\sum_{\substack{f \in D(\Gamma)_{2}, f \subset D(\Omega)}} \kappa(f)=2 \sum_{f \in \Gamma_{2}, f \subset \Omega} \kappa(f)+\sum_{\substack{e \in \Gamma_{1}^{\prime}, e \subset \partial \Omega \cap \partial \Sigma}} 2 \theta(e) .
$$

Finally, $\chi(D(\Omega))=2 \chi(\Omega))-n(\Omega)$ and $m(D(\Omega))=2 m(\Omega)$, and it follows that (3) holds for $\Omega$.

Conversely, suppose that $\Gamma, \kappa$ and $\theta$ satisfy condition (2) of Theorem 1.6, and let $\Omega$ be an admissible domain in $(D(\Sigma), D(\Gamma))$. Then $\Omega$ can be decomposed as the disjoint union of two domains $\Omega_{+}$and $\Omega_{-}$, which are the intersections of $\Omega$ with the two copies of $\Sigma$ which are glued in $D(\Sigma)$. Then both $\Omega_{+}$and $\Omega_{-}$satisfy condition (2) of Theorem 1.11, and the computation made above shows directly that $\Omega$ satisfies condition (2) of Theorem 1.6.

Proof of Theorem 1.10 We can now show that Theorem 1.10 (resp., Theorem 1.11) is a consequence of Theorem 1.4 (resp., Theorem 1.6).

Consider first a framed hyperideal circle pattern $C$ on a compact surface $\Sigma$ with boundary. Let $\Gamma$ be the incidence graph of $C$, and let $\Gamma^{\prime}$ be its extended incidence graph; let $\kappa$ and $\theta$ be the singular curvature and intersection data of $C$, so that $\kappa$ is a function defined on the set of faces of $\Gamma$ and $\theta$ is defined on the set of edges of $\Gamma^{\prime}$. We consider again the doubled surface $D(\Sigma)$, and the doubled circle pattern $D(C)$, which has incidence graph $D(\Gamma)$, as above. By Theorem 1.6, $D(\Sigma)$ and $D(C)$ are uniquely determined by $D(\Gamma)$ and by the intersection angles between the circles and the singular curvature. This already shows that framed hyperideal circle patterns on hyperbolic surfaces are uniquely determined by their incidence graph and intersection angles and singular curvatures data. The same argument can be used for Euclidean circle patterns, based on Theorem 1.4.

To prove the existence of a circle pattern with given incidence graph on a surface with boundary $\Sigma$ and with given intersection angles and singular curvatures, consider the double $D(\Sigma)$ of $\Sigma$, and the graph $D(\Gamma)$ embedded in $D(\Sigma)$, obtained by the construction described above; $D(\Gamma)$ contains two copies of $\Gamma^{\prime}$ which are glued along their boundary faces (each boundary face of one copy is identified with a boundary face of the other copy). There are natural intersection angles $D(\theta)$ and singular curvatures $D(\kappa)$ defined on $D(\Gamma)$ from $\kappa$ and $\theta$ (see above). By Lemma 8.1, Theorem 1.6 can be applied to $(D(\Sigma), D(\Gamma))$ with the singular curvatures and intersection angles data given by $D(\kappa)$ and $D(\theta)$. Thus, the "doubled" data are realized uniquely as incidence graph, intersection angles and singular curvature of a hyperideal circle pattern $D(\Sigma)$ on $D(\Sigma)$.

Moreover, this circle pattern (and the underlying metric) have the same symmetry as $D(\Sigma)$ and $D(\Gamma)$, so $D(\Sigma)$ can be "cut" along a polygonal line to obtain two isometric surfaces with polyhedral boundary, each with a framed hyperideal circle pattern, as required by Theorem 1.11. The same arguments shows Theorem 1.10 from Theorem 1.4. 


\subsection{A Remark and Some Open Questions}

An Alternate Proof when $\kappa$ is Non-negative There is another possible proof of the main theorems, or at least of the infinitesimal rigidity lemmas, which is based on a completely different argument. However this proof works only under the hypothesis that the total angle at the singular points is at most equal to $2 \pi$, i.e. when the singular curvature at the singular points is positive. Moreover it only works for strictly hyperideal circle patterns.

In those cases, the infinitesimal rigidity can be proved by first constructing a hyperbolic cone-manifold with polyhedral boundary associated to a hyperideal circle pattern, as in Sect. 4, and then doubling it, to obtain a complete finite volume conemanifold, which has as singular locus a link (a disjoint union of closed curves). The condition on the angles at the singular points translates as the fact that the total angle around the singular curves is less than $2 \pi$, so that it is possible to apply a rigidity result of Hodgson and Kerckhoff [19], according to which those cone-manifolds cannot be deformed without changing the total angle around some of the singular curves.

It is quite striking that, in those examples, there are two ways to prove the infinitesimal rigidity of those special circle patterns, one based on the volume argument used here, and another based on the more analytic methods used by Hodgson and Kerckhoff [19].

Spherical Metrics All the results presented here concern surfaces endowed with either Euclidean or hyperbolic surfaces with conical singularities. However, the basic result in the theory, the Koebe theorem on circle packings, applies to the sphere. As already pointed out, the result of Bao and Bonahon [3] also translates as a statement on hyperideal circle patterns on the sphere. Interesting recent results of Luo [25] also apply. It would be interesting to know whether results similar to those proved here for Euclidean or hyperbolic metrics holds for circle patterns on surfaces with spherical metrics with conical singularities.

The main problem there appears to be the infinitesimal rigidity of such circle patterns, since the tools used in Sects. 4 and 5 do not appear to work in the spherical context. The compactness results, however, could presumably be proved by the methods used here.

Acknowledgements The results presented here owe much to many fruitful conversations with Boris Springborn, who was strongly involved in parts of the research presented here. I would also like to thank Igor Rivin for several conversations concerning some techniques used in this text, and an anonymous referee for many helpful comments on a previous version of the text.

\section{References}

1. Alexandrov, A.D.: Convex Polyhedra. Springer Monographs in Mathematics. Springer, Berlin (2005). Translated from the 1950 Russian edition by N.S. Dairbekov, S.S. Kutateladze and A.B. Sossinsky, with comments and bibliography by V.A. Zalgaller and appendices by L.A. Shor and Y.A. Volkov

2. Andreev, E.M.: On convex polyhedra of finite volume in Lobacevskii space. Math. USSR Sb. 12(3), 225-259 (1971)

3. Bao, X., Bonahon, F.: Hyperideal polyhedra in hyperbolic 3-space. Bull. Soc. Math. France 130(3), 457-491 (2002) 
4. Bobenko, A.I., Springborn, B.A.: Variational principles for circle patterns and Koebe's theorem. Trans. Am. Math. Soc. 356(2), 659-689 (2004) (electronic)

5. Boileau, M., Leeb, B., Porti, J.: Geometrization of 3-dimensional orbifolds. Ann. Math. (2) 162(1), 195-290 (2005)

6. Boileau, M., Porti, J.: Geometrization of 3-orbifolds of cyclic type. Astérisque 272, 208 (2001). Appendix A by M. Heusener and J. Porti

7. Bonahon, F., Otal, J.-P.: Laminations mesurées de plissage des variétés hyperboliques de dimension 3. Ann. Math. 160, 1013-1055 (2004)

8. Bowditch, B.H.: Singular Euclidean structures on surfaces. J. Lond. Math. Soc. (2) 44(3), 553-565 (1991)

9. Brägger, W.: Kreispackungen und Triangulierungen. Enseign. Math. (2) 38(3-4), 201-217 (1992)

10. Cauchy, A.L.: Sur les polygones et polyèdres, second mémoire. J. Ec. Polytechn. 19, 87-98 (1813)

11. Charney, R., Davis, M.: The polar dual of a convex polyhedral set in hyperbolic space. Mich. Math. J. 42, 479-510 (1995)

12. Colin de Verdière, Y.: Un principe variationnel pour les empilements de cercles. Invent. Math. 104(3), 655-669 (1991)

13. Cooper, D., Hodgson, C.D., Kerckhoff, S.P.: Three-Dimensional Orbifolds and Cone-manifolds. MSJ Memoirs, vol. 5. Mathematical Society of Japan, Tokyo (2000). With a postface by Sadayoshi Kojima

14. Coxeter, H.S.M.: A geometrical background for de Sitter's world. Am. Math. Mon. 50, 217-228 (1943)

15. Edelsbrunner, H.: Geometry and Topology for Mesh Generation. Cambridge Monographs on Applied and Computational Mathematics, vol. 7. Cambridge University Press, Cambridge (2001)

16. Gallot, S., Hulin, D., Lafontaine, J.: Riemannian Geometry, 3rd edn. Universitext. Springer, Berlin (2004)

17. Garrett, B.T.: Circle packings and polyhedral surfaces. Discrete Comput. Geom. 8(4), 429-440 (1992)

18. Gel'fand, I.M., Graev, M.I., Vilenkin, N.J.: Obobshchennye funktsii, Vyp. 5. Integralnaya geometriya i svyazannye s nei voprosy teorii predstavlenii. Gosudarstv. Izdat. Fiz.-Mat. Lit., Moscow (1962)

19. Hodgson, C.D., Kerckhoff, S.P.: Rigidity of hyperbolic cone-manifolds and hyperbolic Dehn surgery. J. Differ. Geom. 48, 1-60 (1998)

20. Kharevych, L., Springborn, B., Schröder, P.: Discrete conformal mappings via circle patterns. ACM Trans. Graph. 25(2), (2006)

21. Labourie, F., Schlenker, J.-M.: Surfaces convexes fuchsiennes dans les espaces lorentziens à courbure constante. Math. Ann. 316, 465-483 (2000)

22. Legendre, A.-M.: Eléments de géométrie. Paris (1793) (an II). Première édition, note XII, pp. 321334

23. Leibon, G.: Characterizing the Delaunay decompositions of compact hyperbolic surfaces. Geom. Topol. 6, 361-391 (2002) (electronic)

24. Leibon, G.: Random Delaunay triangulations and metric uniformization. Int. Math. Res. Not. 25, 1331-1345 (2002)

25. Luo, F.: A characterization of spherical polyhedron surfaces. J. Differ. Geom. 74(3), 407-424 (2006)

26. Milnor, J.: The Schläfli differential equality. In: Collected Papers, vol. 1. Publish or Perish (1994)

27. Otal, J.-P.: Sur le cœur convexe d'une variété hyperbolique de dimension 3. Preprint (1994)

28. Pogorelov, A.V.: Extrinsic Geometry of Convex Surfaces. Translations of Mathematical Monographs, vol. 35. American Mathematical Society, Providence (1973)

29. Rivin, I.: On the geometry of convex polyhedra in hyperbolic 3-space. Ph.D. thesis, Princeton University (1986)

30. Rivin, I.: Euclidean structures on simplicial surfaces and hyperbolic volume. Ann. Math. 139, 553580 (1994)

31. Rivin, I.: A characterization of ideal polyhedra in hyperbolic 3-space. Ann. Math. 143, 51-70 (1996)

32. Rivin, I.: Combinatorial optimization in geometry. Adv. Appl. Math. 31(1), 242-271 (2003)

33. Rivin, I., Hodgson, C.D.: A characterization of compact convex polyhedra in hyperbolic 3-space. Invent. Math. 111, 77-111 (1993)

34. Rousset, M.: Sur la rigidité de polyèdres hyperboliques en dimension 3 : cas de volume fini, cas hyperidéal, cas fuchsien. Bull. Soc. Math. France 132, 233-261 (2004)

35. Sabitov, I.K.: Around the proof of the Legendre-Cauchy lemma on convex polygons. Sib. Mat. Zh. 45(4), 892-919 (2004). Translated in Sib. Math. J. 45(4), 740-762 (2004)

36. Schlenker, J.-M.: Métriques sur les polyèdres hyperboliques convexes. J. Differ. Geom. 48(2), 323405 (1998)

37. Schlenker, J.-M.: Convex polyhedra in Lorentzian space-forms. Asian J. Math. 5(2), 327-363 (2001) 
38. Schlenker, J.-M.: Hyperbolic manifolds with polyhedral boundary. math.GT/0111136, available at http://picard.ups-tlse.fr/ schlenker (2001)

39. Schlenker, J.-M.: Hyperideal polyhedra in hyperbolic manifolds. Preprint math.GT/0212355 (2002)

40. Schlenker, J.-M.: Hyperideal circle patterns. Math. Res. Lett. 12(1), 82-112 (2005). math.GT/0407043

41. Schlenker, J.-M.: A rigidity criterion for non-convex polyhedra. Discrete Comput. Geom. 33(2), 207221 (2005). math.DG/0301333

42. Schlenker, J.-M.: Small deformations of polygons and polyhedra. Trans. Am. Math. Soc. 359, 21552189 (2007)

43. Springborn, B.A.: A variational principle for weighted Delaunay triangulations and hyperideal polyhedra. J. Differ. Geom. (2006, to appear). arXiv:math.GT/0603097

44. Thurston, W.P.: Three-dimensional geometry and topology. Recent version available on http://www. msri.org/publications/books/gt3m/ (1980) 